\title{
Spektrale Geometrie und Huygenssches Prinzip für Tensorfelder und Differentialformen I
}

\author{
R. Schmmmina
}

Es werden verschiedene Laplaceoperatoren $L$ zu ciner $n$-dimensionalen Riemannschen Mannigfaltigkeit $(M, g)$ betrachtet, die auf Tensorfelder bzw. (alternierende bzw. symmetrische) Differentialformen $\boldsymbol{p}$-ter Stufe wirken. Für definites $\boldsymbol{g}$ und geschlossenes $\boldsymbol{M}$ werden Ergebnisse zur spektralen Geometrie erhalten. Ist z. B. ein gewisses $L$. isospektral zum Laplacian einer flachen Mannigfaltigkeit und $p$ größer als eine von $n$ abhängige Schranke, so ist $(\dot{M}, g)$ flach. Für lorentzsches $g$ und $n=6$ werden Ergebnisse zum Huygensschen Prinzip $(H P)$ erhalten. Aus dem $H P$ und gewissen Zusatzannahmen folgt, daB $(M, g)$ flach ist.

Рассматриваются разные операторы Лапласа $L$, действующие на тензорные поля или на дифференциальные формы (альтернируюцие или симметрические) на $n$-мерном римановом многообразии $(M, g)$. Для знакоопреденной $g$ и замкнутого $M$ получены результаты по спектральной геометрии. Если, например, некоторыи $L$ изоспектрально к лапласиану плоского мкогообразия и степень $p$ больше границы, зависящейся от $n$, то $(M, g)$ является плоским. Для лорснцовой $g$ и $n=6$ получены результаты по принұипе Гюйгена (ПГ). При допольнтельных предположениях из $П Г$ следует, что (M; g) является плоскнм.

Several Laplace operators $L$ over an $n$-dimensional Riemannian manifold ( $M, g$ ), applying to tensor fields and (alternating or symmetric) differential forms of degree $p$, are considered. For definite $g$ and closed $\boldsymbol{M}$ results on spectral geometry are obtained. If e.g. some $L$ is isospectral to a Laplacian of a flat manifold and $p$ greater than some bound depending on $n$, then $(\boldsymbol{M}, g)$ is flat. For lorentzian $g$ and $n=6$ results on Huygens' principle $(H P)$ are obtained. From $H P$ and additional assumptions there follows that $(M, g)$ is flat.

\section{Einleitung}

Es werden die folgenden Strukturen über einer orientierten Mannigfaltigkeit der Dimension $n \geqq 2$ betrachtet:

- Eine definite oder lorentzsche riemannsche Metrik

$$
g=g_{\alpha \beta} d x^{\alpha} d x^{\beta} \quad(\alpha, \beta=1,2, \ldots, n) .
$$

- Die Vektorbündel

$$
\otimes^{p} T^{*}, \quad A^{p} T^{*} \quad(0 \leqq p \leqq n), \quad \Pi^{p} T, \quad \Pi^{p} T^{*} / g,
$$

welche aus.allen $p$-stufigen Tensoren bzw. nur aus den alternierenden Tensoren bzw. den symmetrischen Tensoren bzw. den bezüglich $g$ spurfreien symmetrischen Tensoren bestehen.

- Die Laplaceoperatoren oder Laplacians

$$
\Delta:=g^{\alpha \beta} \nabla_{\alpha}^{\prime} \nabla_{\beta} \text { mit } \nabla:=\text { Levi-Civita-Ableitung zu } g,
$$




$$
\begin{aligned}
& \Delta-\frac{n-2}{4(n-1)} R I \text { mit } I:=\text { identischer Operator, } \\
& \varepsilon \delta d-d \delta \equiv \Delta+\varepsilon Z \text { mit dem Indikator } \varepsilon:=\left\{\begin{array}{l}
-1 \text { für } \Lambda^{p} T^{*} \\
+1 \text { für } \Pi^{p} T^{*}
\end{array}\right.
\end{aligned}
$$

und dem aus dem Krümmungstensor gebildeten Weitzenböck-Operator $Z$.

Wir verwenden folgende Sammelbezeichnungen:

$$
\begin{aligned}
& E^{p}:=\bigotimes^{p} T^{*}, \Lambda^{p} T^{*}, \quad \Pi^{p} T^{*}, \Pi \Pi^{p} T^{*} / g \\
& E:=\underset{p \geqq 0}{\bigotimes} E^{p}=: \bigotimes T^{*}, \quad \Lambda T^{*}, \Pi T^{*}, \Pi T^{*} / g \\
& L=\Delta+C \operatorname{mit} C=0,-\frac{n-2}{4(n-1)} R I, \varepsilon Z ; \\
& \mathfrak{L}:=C+\frac{n-2}{4(n-1)} R I=\text { Cottoninvariante von } L .
\end{aligned}
$$

Der „reine Lapalacian" $\Delta$ und der Laplacian mit $\mathfrak{L}=\mathbf{0}$ wirken auf die Schnitte eines beliebig gewählten $E^{p}$. Der aus

$$
\text { …... } \quad d:=\text { äußeres Differential, } \delta:=\text { inneres Differential }
$$

gebildete „kanonische Laplacian“ $\varepsilon \delta d-d \delta$ wirkt dagegen nur auf (alternierende öder symmetrische) Differentialformen. Alle drei Typen von Laplacians $L=\Delta+C$ sind in der physikalischen Literatur anzutreffen! Griechische Indizes werden wio gewohnt mittels $\left(g_{\alpha \beta}\right)$ gesenkt bzw. mittels $\left(g^{\alpha \beta}\right):=\left(g_{\alpha \beta}\right)^{-1}$ gehoben. Weiter bezeichnen wir:

$$
\begin{aligned}
& \langle u, v\rangle:=\text { Skalarprodukt bezüglich } g \text { von Tensoren } u, v ; \\
& |u|^{2}:=\langle u, u\rangle ; \\
& \|u\|^{2}:=\int_{\mu}|u|^{2} d \text { Vol für geschlossenes } M ; \\
& T r:=\text { Spur bezüglich } g \text { und } E^{p} ; \\
& \text { Riem }=R_{\alpha \beta \mu \nu} d x^{\alpha} \wedge d x^{\beta} d x^{\mu} \wedge d x^{\gamma}=\text { Krümmungstensor; } \\
& \text { Ric }=R_{\alpha \beta} d x^{\alpha} d x^{\beta}:=g^{\mu \nu} R_{\alpha \mu \gamma \beta} d x^{\alpha} d x^{\beta}=\text { Riccitensor; } \\
& R:=g^{\alpha \beta} R_{\alpha \beta}=S k a l a r k r u ̈ m m u n g ; \\
& W e y l=C_{\alpha \beta \mu \nu} d x^{\alpha} \wedge d x^{\beta} d x^{\mu} \wedge d x^{\gamma}=\text { Konformkrümmungstensor; } \\
& S=S_{\alpha \beta} d x^{\alpha} d x^{\beta}:=R i c-\frac{1}{n} R \cdot g .
\end{aligned}
$$

Nach J. Hadamard [9] kann im Falle der Analytizität die Grundlösung von $L$ aus Potenzreihen in

$$
\sigma=\sigma(x, y)=\frac{1}{2}(\text { geodätischer Abstand von } x, y)^{2}
$$

aufgebaut werden. Die Entwicklungskoeffizienten sind dabei proportional zu den sogenannten Hadamardkoeffizienten $U_{k}=U_{k}(x, y)(k=0,1,2, \ldots)$, welche man auch für den Fall $C^{\infty}$ erklären kann. Ab.jetzt seien alle betrachteten Objekte von der Klasse $C^{\infty}$. Aus den $U_{k}$ kann man Informationen über $L$ und dadurch vermittelt auch über $M, g, E^{p}$ gewinnen, insbesondere in den beiden Situationen

(I) $g$ definit, $M$ geschlossen (:= kompakt, randlos, zusammenhängend);

(II) $g$ lorentzsch, $n=: 2 m+2 \geqq 4$ geradzahlig. 
In der vorliegenden Arbeit werden Informationen speziell aus $U_{2}(x, x)$ gewonnen. Die Spur (im Sinne der Spur eines linearen Operators) $\operatorname{Tr} U_{2}(x, x)$ ist eine Linearkombination in $\mid$ Riem $\left.\right|^{2}$, $|R i c|^{2}, R^{2}, \Delta R$; deshalb kann man unter Umständen von $\operatorname{Tr} U_{2}$ auf die Krümmung Riem schließen.

Spektrale Geometrie: Bei (I) besitzt der formal selbstadjungierte elliptische Differentialoperator $L$ eine Folge von reellen Eigenwerten endlicher Vielfach heit als Spektrum. Man fragt dann nach den Eigenschaften von $(M, g)$, die sich im Spektrum von $L$ widerspiegeln. Die Zahlen

$$
U_{k}(M):=\int_{M} \operatorname{Tr} U_{k}(x, x) d \operatorname{Vol}(x) \quad(k=0,1,2, \ldots)
$$

sind spektrale Invarianten. Ist $L$ isospektral zum Laplacian einer flachen Mannigfaltigkeit, so gilt folglich $U_{k}(M)=0$ für $k \geqq 1$. Durch Auswertung von $U_{2}(M)=0$ beweisen wir unter anderem:

1.-Ist L isospektral zum Laplacian einer flachen Mannigfaltigkeit und ist 48p größer oder gleich der aus der Tabelle

\begin{tabular}{l|l|l}
\hline $\begin{array}{l}E= \\
+\end{array}$ & $\Delta=+$ & $\Delta-\frac{n-2}{4(n-1)} R I$ \\
\hline$\otimes T^{*}$ & $4 n^{3}$ & $n^{3}$ \\
\hline$\Pi T^{*}$ & $8 \sqrt{3} n^{2}$ & $4 \sqrt{3} n^{2}$ \\
\hline$\Pi T^{*} / g$ & $8 \sqrt{3} n^{2}$ & $4 \sqrt{3} n^{2}$ \\
\hline
\end{tabular}

entnommene Schranke, so ist $(M, g)$ flach.

(Wir werden auch noch feinere Abschätzungen für $p$ herleiten! Die Aussage beziebt sich nur auf die in der Tabelle erfaßten Typen von $L$ und $E^{p}$.)

2. Ist $\varepsilon \delta d-d \delta$ für $n \geqq 4$ und drei paarweise verschiedene Stufen $p$ isospektral zum Laplacian einer flachen $\bar{M}$ annigfaltigkeit, so ist $(M, g)$ flach.

Die in die spektrale Geometrie geschlossener Mannigfaltigkeiten hier neu eingebrachten Gesichtspunkte sind insbesondere die Betrachtung

- nicht nur alternierender Differentialformen, sondern auch symmetrischer Differentialformen, spurfrei-symmetrischer Differentialformen und kovarianter Tensorfelder;

- nicht nur des kanonischen Laplacians, sondern auch gewisser „,nichtkanonischer“ Laplacians.

Im übrigen verweisen wir auf die Literatur, insbesondere $[2,13,11,4,5,8]$.

Huygenssches Prinzip (abgekürzt: $H P$ ): Bei (I) kann man fragen, ob der hyperbolische Differentialoperator $L$ dem $H P$ im Sinne von [3] geniigt. Das Hadamardsche Kriterium besagt: $L$ ist huygenssch genau dann, wenn

$$
\sigma(x, y)=0 \Rightarrow U_{m}(x, y)=0
$$

für hinreichend benachbarte Punkte $x, y \in M$. Im physikalischen Fall $n=4$ kann man das $H P$ so interpretieren, da $\beta$ mit den Lösungen $u=u(x)$ der ,Wellengleichung، $L[u]=0$ eine ungestörte (d. h. ohne Nacheffekte, ohne Schweifterme) Signaliibertragung möglich ist. Wir beschränken uns auf den Fall $n=6$. Durch Auswertung von $\operatorname{Tr} U_{2}(x, x)=0$ beweisen wir unter anderem: 
Es seien $|S|^{2}, \mid$ Weyl $\left.\right|^{2}$ positiv definit und $\operatorname{Tr} \Delta \mathfrak{L} \leqq 0$ und $p$ größer oder gleich der aus der Tabelle

\begin{tabular}{|c|c|c|}
\hline$\underset{t}{E}=\quad L=\rightarrow$ & $\Delta$ & $\Delta-\frac{1}{5} R I$ \\
\hline$\otimes T^{*}$ & 15 & 1 \\
\hline$\Pi T^{*}$ & 8 & 1 \\
\hline$\Pi T^{*} / g$ & 7 & 1 \\
\hline
\end{tabular}

entnommene Schranke. Wenn dann $L$ dem $H P$ genügt, so ist $(M, g)$ flach. (Wir werden einfache Beispiele dafür konstruieren, wie man die obigen Voraussetzungen befriedigen und die Schranke für $p$ teilweise noch verkleinern kann. Die Aussage bezieht sich nur auf die in der Tabelle erfaßten Typen von $L$ und $E^{p}$.) Die vorliegende Arbeit setzt die Untersuchungen des Autors zum $H P$ bei $n=6$ fort $[20,17]$.

\section{§ 1. Natürliche Projektoren}

Es sind natürliche Injektionen

$$
\Lambda^{p} T^{*} \bigcirc \otimes p T^{*} \bigcirc \Pi^{p} T^{*} \bigcirc \Pi^{p} T^{*} / g
$$

definiert und in jeweils umgekehrter Richtung natürliche Projektionen. Entsprechend hat man natürliche Projektoren

$$
\text { . } P=P^{(p)}: \otimes{ }^{p} T^{*} \rightarrow E^{p}
$$

und $\{\ldots\}$ bezeichne die jeweils zugehörige ,Indexoperation“". Für $E^{p}=\Lambda^{p} T^{*}$ ist \{...\} gleich der Alternierung [...] und für $E^{p}=\Pi^{p} T^{*}$ gleich der Mischung (...). In Komponenten kann man für $p \geqq 2$ schreiben

$$
\begin{aligned}
& (P u)_{\alpha_{1} \alpha_{2} \ldots \alpha_{p}}=P_{\alpha_{1} \alpha_{1} \ldots \alpha_{p}}^{\beta_{1} \beta_{1} \ldots \beta_{p}} u_{\beta_{1} \beta_{2} \ldots \beta_{p}}=u_{\left\{\alpha_{1} \alpha_{2} \ldots \alpha_{p}\right\}}, \\
& P_{\alpha_{1} \alpha_{2} \ldots \alpha_{p}}^{\beta_{2} \beta_{2} \ldots \beta_{p}}=\delta_{\left\{\alpha_{1}\right.}^{\beta_{1}} \delta_{\alpha_{z}}^{\beta_{2}} \ldots \delta_{\alpha_{p}}^{\left.\beta_{p}\right\}} .
\end{aligned}
$$

Für $p=0$ und $p=1$ werden die vier Bündel (1.1) identifiziert und

$$
P^{(0)}=1, \quad P_{a}^{\beta}=\delta_{a}^{\beta}
$$

gesetzt. Die Komponenten $P_{a_{1} \alpha_{3}}^{\beta_{1} \beta_{2}}$ von $P^{(2)}$ sind gleich

$$
\delta_{a_{1}}^{\beta_{2}} \delta_{a_{2}}^{\beta_{2}}, \delta_{\left[\alpha_{1}\right.}^{\beta_{1}} \delta_{\left.a_{2}\right]}^{\beta_{2}}, \delta_{\left(a_{1}\right.}^{\beta_{1}} \delta_{\left.a_{2}\right)}^{\beta_{1}}, \delta_{\left(a_{2}\right.}^{\beta_{1}} \delta_{\left.a_{2}\right)}^{\beta_{2}}-\frac{1}{n} g_{a_{1} \alpha_{3}} g^{\beta_{1} \beta}
$$

beziehentlich für $\otimes T^{*}, \Lambda T^{*}, \Pi T^{*}, \Pi T^{*} / g$. Wir setzen ferner

$$
P_{\alpha_{1} \alpha_{3}, \beta_{1} \beta_{2}}:=g_{\beta_{1} \mu_{1}} g_{\beta_{3,} \mu_{1}} P_{\alpha_{2} \alpha_{z}}^{\mu_{1} \mu_{3}} \text {. }
$$

Satz 1.1: Aus $g_{\alpha \beta} A^{\alpha \beta}=0$ folgt

$$
2 P_{\alpha \mu, \beta v} A^{\mu v}=\gamma A_{(\alpha \beta)}-\varepsilon A_{\{\alpha \beta\}}
$$

mit beziehentlich in den vier Fällen

$$
\gamma:=0,-1,+1,1-\frac{2}{n}, \quad \varepsilon:=0,-1,+1,1+\frac{2}{n} .
$$


Wir definieren:

$$
N_{0}^{p}:=\text { Faserdimension von } E^{p}, N_{q}^{p}:=\left(\begin{array}{l}
p \\
q
\end{array}\right) N_{0} p / N_{0}^{q}
$$

und betreiben mit diesen Zahlen etwas „Kombinatorik“. .

Satz 1.2: Es gilt beziehentlich in den vier Fällen

$$
\begin{aligned}
& N_{0}^{p}=n^{p},\left(\begin{array}{l}
n \\
p
\end{array}\right), \quad\left(\begin{array}{c}
n+p-1 \\
p
\end{array}\right), \quad\left(\begin{array}{c}
n+p-1 \\
p
\end{array}\right)-\left(\begin{array}{c}
n+p-3 \\
p-2
\end{array}\right) ; \\
& N_{q}^{p}=\left(\begin{array}{l}
p \\
q
\end{array}\right) n^{p-q},\left(\begin{array}{c}
n-q \\
p-q
\end{array}\right),\left(\begin{array}{c}
n+p-1 \\
p-q
\end{array}\right), \frac{n+2 p-2}{n+2 q-2}\left(\begin{array}{c}
n+p-3 \\
p-q
\end{array}\right) \quad(n+q \geqq 3) .
\end{aligned}
$$

Für $E=\Lambda T^{*} b z w .=\Pi T^{*}$ gilt die Formel

$$
\sum_{q=0}^{r} \varepsilon^{q}\left(\begin{array}{l}
r \\
q
\end{array}\right) N_{q+r}^{p}=N_{2 r}^{p+r}
$$

Den Beweis von (1.4) führt man am besten für die beiden Fälle gesondert durch vollständige Induktion über $r$. Für $\Lambda T^{*}$ findet sich die Formel (1.4) bereits in [8]; neu ist ihre Utbertragung auf $\Pi T^{*}$

In der Algebra der symmetrischen Differentialformen mögen · bzw. $\lrcorner$ die äußere bzw. innere Multiplikation bezeichnen und $e^{\alpha}$ bzw. $i_{\alpha}$ die durch

$$
\begin{aligned}
& e^{\alpha} u_{p}:=d x^{\alpha} \cdot u_{p}, \\
& i_{\alpha} u_{p}:=u_{\alpha \alpha_{\alpha} \ldots a_{p}} d x^{\alpha_{2}} \ldots d x^{\alpha_{p}} \quad(p \geqq 1), i_{\alpha} u_{0}:=0 \quad(p=0)
\end{aligned}
$$

definierte äußere bzw. innere Indexoperation sowie

$$
g^{p}:=g \cdot g \cdot \cdots \cdot g \quad(p \text {-mal }) .
$$

Satz 1.3: Die natürliche Projektion $\Pi^{p} T^{*} \rightarrow \Pi^{p} T^{*} / g$ ist gleich

$$
\sum_{q=0}^{[p / 2]} a_{q}^{p} G_{q}^{p}
$$

mit Koeffizienten $a_{q}^{p}$ gemä $\beta$

$$
(-4)^{q}\left(\begin{array}{c}
n / 2+p-2 \\
q
\end{array}\right) a_{q}^{p}:=\left(\begin{array}{c}
p \\
2 q
\end{array}\right)\left(\begin{array}{c}
2 q \\
q
\end{array}\right)
$$

und linearen Operatoren $G_{q}{ }^{p}$ gemä $\beta$

$$
\left.G_{q}^{p} u_{p}:=g^{q} \cdot\left(g^{q}\right\lrcorner u_{p}\right) .
$$

Äquivalent zu (1.6) ist die Rekursionsformel (in der sich $N_{p-1}^{p}$ auf $\Pi T^{*} / g$ beziehe)

$$
(p-2 q)(n+p+2 q-1) a_{q}^{p}+4(q+1)^{2} a_{q+1}^{p}=p N_{p-1}^{q} a_{q}^{p-1} .
$$

Es gilt des weiteren die Formel

$$
p^{2} i_{a} G_{q} p^{a} e^{a}=(p-2 q)(n+p+2 q-1) G_{q}^{p-1}+4 q^{2} G_{q-1}^{p-1} .
$$

Den Beweis von (1.9) geben wir hier nicht an. Die übrigen Behauptungen wurden in [18] bewiesen.

Satz 1.4: Es gilt für $p \geqq 2$ und $1 \leqq k \leqq p-1$

$$
N_{0}{ }^{k} P_{\beta_{1} \ldots \beta_{k} \alpha_{k+1} \ldots a_{p}}^{a_{1} \ldots \alpha_{k} \alpha_{k+1} \ldots \alpha_{p}}=N_{0}^{p} P_{\beta_{1} \ldots \beta_{k}}^{\alpha_{1} \ldots \alpha_{k}}
$$


Beweis: Es geniigt, (1.10) für $k=p-1$ zu zeigen, d. h.

$$
P_{\alpha \beta_{2} \ldots \beta_{p}}^{a \alpha_{2} \ldots \alpha_{p}}=\frac{N_{0}^{p}}{N_{0}^{p-1}} P_{\beta_{3} \ldots \beta_{p}}^{\alpha_{3} \ldots \alpha_{p}}
$$

Die allgemeine Formel (1.10) ergibt sich dann durch iterierte Anwendung von (1.11). Der Beweis von (1.11) verläuft nun unterschiedlich für die vier Fälle:

$\otimes T^{*}:$ Trivial.

$\Lambda T^{*}$ und $\Pi \dot{T}^{*}:$ Aus der Entwicklungsformel

$$
p \cdot P_{\beta \beta_{1} \ldots \beta_{p}}^{\alpha \alpha_{1} \ldots \alpha_{p}}=\delta_{\beta}^{\alpha} P_{\beta_{3} \ldots \beta_{p}}^{\alpha_{1} \ldots \alpha_{p}}+\varepsilon \sum_{k=2}^{p} \delta_{\beta_{k}}^{\alpha} P_{\beta_{2} \ldots \beta_{k} \ldots \beta_{p}}^{\alpha_{\alpha} \ldots \ldots \beta_{p}}
$$

erhalten wir durch Verjüngung

$$
p \cdot P_{\alpha \beta_{2} \ldots \beta_{p}}^{\alpha \alpha_{2} \ldots \alpha_{p}}=[n+\varepsilon(p-1)] P_{\beta_{2} \ldots \beta_{p}}^{\alpha_{2} \ldots \alpha_{p}}=N_{p-1}^{p} P_{\beta_{2} \ldots \beta_{p}}^{\alpha_{1} \ldots \alpha_{p}}
$$

In (1.12) und im folgenden kennzeichne das Symbol $\wedge$ eine Lücke in einer Indexfolge. Ein Index unter $\wedge$ ist sinngemä $B$ in die Lücke einzusetzen.

$\Pi^{p} T^{*} / g$ : Indem wir der Einfachheit halber die Mischung - d. h. die natürliche Projektion $\otimes T^{*} \rightarrow \Pi T^{*}-$ als rechtsseitigen Faktor in der nachfolgend angedeuteten Rechnung mit Operatoren unterdrücken, erhalten wir aus den Formeln (1.8) und (1.9)

$$
p^{2} i_{\alpha} P^{(p)} e^{\alpha}=\sum_{q=0}^{[p / 2]} a_{q}^{p} p^{2} i_{\alpha} G_{q}^{p} e^{\alpha}=\cdots=p N_{p-1}^{p} P^{(p-1)}
$$

Die Komponenten von $i_{a} P^{(p)} e^{a}$ sind aber gerade die linke Seite von (1.11)

\section{Laplacians und ihre Hadamardkoeffizienten}

Die Ricciidentität für Tensorfelder schreiben wir als

$$
\begin{aligned}
& \left(\nabla_{\alpha} \nabla_{\beta}-\nabla_{\beta} \nabla_{\alpha}\right) u=K_{\alpha \beta} u, \\
& \left(K_{\alpha \beta} u\right)_{\alpha_{1} \alpha_{1} \ldots \alpha_{p}}=\sum_{k=1}^{p} R_{\alpha \beta \alpha_{k}}{ }^{\mu} \cdot u_{\alpha_{1} \ldots \alpha_{k} \ldots \alpha_{p}} \quad(p \geqq 1) .
\end{aligned}
$$

Satz 2.1: Der kanonische Laplacian besitzt die Darstellung

$$
\varepsilon \delta d-d \delta=\Delta+\varepsilon Z
$$

mit einem linearen Operator $Z$ gemä $\beta$

$$
\begin{aligned}
& (Z u)_{\alpha_{1} \alpha_{2} \ldots \alpha_{p}}=\sum_{k=1}^{p} R_{\alpha_{k}}^{\mu} u_{\alpha_{1} \ldots \alpha_{k} \ldots \alpha_{p}} \\
& -2 \sum_{k=1}^{p-1} \sum_{l=k+1}^{p} R_{\alpha_{k} \alpha_{l} \cdot}^{p} u_{\alpha_{1} \ldots \alpha_{k} \ldots \alpha_{j} \ldots \alpha_{z}} \quad(p \geqq 2) \\
& Z u_{0}=0 \quad(p=0), \quad(Z u)_{\alpha}=R_{\alpha}{ }^{\mu} u_{\mu} \quad(p=1) .
\end{aligned}
$$

Beweis: Aus den Darstellungen

$$
\begin{aligned}
(p+1)(d u)_{\alpha \alpha_{1} \ldots \alpha_{p}} & =(p+1) \nabla_{\{\alpha} u_{\left.\alpha_{1} \ldots \alpha_{p}\right\}}=\nabla_{a} u_{\alpha_{1} \ldots \alpha_{p}}+\varepsilon \sum_{k=1}^{p} \nabla_{\alpha_{k}} u_{\alpha_{2} \ldots \alpha_{k} \ldots \alpha_{p}}, \\
(\delta u)_{\alpha_{k} \ldots \alpha_{p}} & =\varepsilon p \nabla^{a} u_{\alpha \alpha_{2} \ldots \alpha_{p}}
\end{aligned}
$$


erhalten wir einerseits

$$
\varepsilon(\delta d u)_{a_{k} \ldots \alpha_{p}}=\Delta u_{a_{1} \ldots \alpha_{p}}+\varepsilon \sum_{k=1}^{p} \nabla^{a} \nabla_{a_{k}} u_{a_{1} \ldots \alpha_{k} \ldots \alpha_{p}}
$$

und setzen hier ein

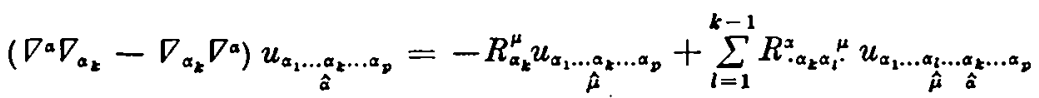

$$
\begin{aligned}
& +\sum_{l=k+1}^{p} R_{\cdot \alpha_{k} \alpha_{l}^{\mu}}^{\mu} \cdot u_{\alpha_{1} \ldots \alpha_{k} \ldots \alpha_{l} \ldots \alpha_{p}} \text {. }
\end{aligned}
$$

Andererseits erhalten wir

$(d \delta u)_{\alpha_{1} \ldots \alpha_{p}}=\frac{1}{p} \sum_{k=1}^{p} \varepsilon^{k+1} \nabla_{\alpha_{k}}(\delta u)_{\alpha_{1} \ldots \alpha_{k} \ldots \alpha_{p}}=\sum_{k=1}^{p} \varepsilon^{k} \nabla_{\alpha_{k}} \nabla^{a} u_{\alpha \alpha_{1} \ldots \alpha_{k} \ldots \alpha_{p}}=\varepsilon \sum_{k=1}^{p} \nabla_{\alpha_{k}} \nabla^{a} u_{\alpha_{1} \ldots \alpha_{k} \ldots \alpha_{p}}$

und daraus die Behauptung $\varepsilon \delta d=\Delta+d \delta+\varepsilon Z$.

Für $\Lambda T^{*}$ ist die Darstellung (2.3) wohlbekannt (Weitzenböck-Formel); neu ist hier die Ưbertragung auf $\Pi T^{*}$ und die Möglichkeit einer einheitlichen Herleitung.

Neben Einpunkt-Größen (Skalare, Tensoren, Differentialformen, ...), welche über der ganzen Mannigfaltigkeit $M$ erklärt sein sollen, sind auch Zweipunkt-Größen von Interesse, welche nur in einer Umgebung der Diagonale von $M \times M$ erklärt zu sein brauchen. Die Einschränkung auf die Diagonale wird als Koinzidenzwert der Zweipunkt-Größe bezeichnet. Die Relation der Gleichheit der Koinzidenzwerte symboli-

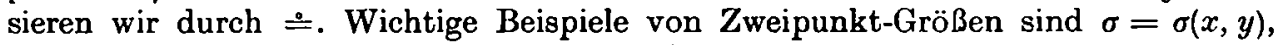
welches durch das Differentialgleichungsproblem



bestimmt wird, sowie die Hadamardkoeffizienten $U_{k}=U_{k}(x, y)$. Letztere kann man als die regulären Lösungen des rekursiven Differentialgleichungssystems

$\sigma^{a} \nabla_{\alpha} U_{0}+\mu U_{0}=0, \quad \sigma^{a} \nabla_{\alpha} U_{k}+(\mu+k) U_{k}=L\left[U_{k-1}\right] \quad(k=1,2, \ldots)$,

nebst der Anfangsbedingung $U_{0} \doteq I$, definieren, welche sich bezüglich des ersten Arguments $x$ wie Schnitte von $E^{p}$ und bezüglich des zweiten Arguments $y$ wie Schnitte des dualen Bündels $E^{p *}$ verhalten. Abkürzend wurde

$$
\sigma^{\alpha}:=g^{\alpha \beta} \nabla_{\beta} \sigma, \quad 2 \mu:=\Delta \sigma-n
$$

gesetzt und die Differentialoperatoren beziehen sich jeweils auf das erste Argument $x$.

Der uns hier interessierende Koinzidenzwert des 2. Hadamardkoeffizienten ist sogar für allgemeinere Laplacians

$$
L=\Delta+2 A^{\star} \nabla_{a}+C
$$

bekannt: P. B. GHKKY $[4,5]$ hat $D_{2}(x, x)$ mittels einer invariantentheoretischen Methode berechnet. Der Autor der vorliegenden Arbeit hat später unabhängig davon das Resultat mittels der "Methode der Koinzidenzwerte“ reproduziert [20, 17]. Einige Spezialfälle sind schon länger bekannt, siehe z. B. $[6,11,2,13]$.

Satz 2.2: Für einen Laplacian $L=\Delta+C$ gilt

$$
\begin{aligned}
180 U_{2} \doteq & \left(R_{\alpha \beta \mu \nu} R^{\alpha \beta \mu \nu}-R_{\alpha \beta} R^{\alpha \beta}\right) I+15 K_{\alpha \beta} K^{\alpha \beta} \\
& +90\left(C+\frac{1}{6} R I\right)^{2}+30 \Delta\left(C+\frac{1}{5} R I\right) .
\end{aligned}
$$




\section{§ 3. Quadratische Krümmungsinvarianten}

Wir betrachten skalare Invarianten der Metrik $g$ der Gestalt

$$
\begin{aligned}
F=F[g] & =a_{2} R_{\alpha \beta \mu \nu} R^{\alpha \beta \mu \nu}+a_{1} R_{\alpha \beta} R^{\alpha \beta}+a_{0} R^{2} \\
& \equiv a_{2}|R i e m|^{2}+a_{1}|R i c|^{2}+a_{0} R^{2}
\end{aligned}
$$

mit $a_{2}, a_{1}, a_{0}=$ const.

Satz 3.1: Die Invariante $F$ ist gleich

$$
\begin{aligned}
& \frac{b_{2}}{4}|W e y l|^{2}+\frac{b_{1}}{n-2}|S|^{2}+\frac{b_{0}}{2 n(n-1)} R^{2} \quad \text { für } n \geqq 4, \\
& b_{1}|S|^{2}+\frac{b_{0}}{12} R^{2} \quad \text { für } n=3, \quad \frac{b_{0}}{4} R^{2} \quad \text { für } n=2,
\end{aligned}
$$

mit den Koeffizienten

$$
\begin{aligned}
& b_{2}:=4 a_{2}, \quad b_{1}:=4 a_{2}+(n-2) a_{1}, \\
& b_{0}:=4 a_{2}+2(n-1)\left(a_{1}+n a_{0}\right) .
\end{aligned}
$$

Definitheitseigenschaften von $F$ lassen sich giunstiger in der Darstellung (3.2) als in der Darstellung (3.1) studieren.

Satz 3.2: 1st die Metrik $g$ definit und ist eine der Bedingungen

$$
\begin{array}{lll}
n \geqq 4 & \text { und } & \operatorname{sgn} b_{2}=\operatorname{sgn} b_{1}=\operatorname{sgn} b_{0} \neq 0, \\
n=3 & \text { und } & \operatorname{sgn} b_{1}=\operatorname{sgn} b_{0} \neq 0, \\
n=2 & \text { und } & b_{0} \neq 0
\end{array}
$$

erfüllt, so ist F eine definite quadratische Form im Krümmungstensor. Genauer hat man:

$F$ positiv definit $\Leftrightarrow \operatorname{Min} b_{k}>0$,

$F$ negativ definit $\Leftrightarrow \operatorname{Max} b_{k}<0$.

Wir haben gesetzt sgn $=$ signum $=$ Vorzeichen. Zur Bestimmung des minimalen und des maximalen $b_{k}$ sind die Formeln nützlich

$$
\begin{aligned}
& b_{1}-b_{2}=(n-2) a_{1}, \quad b_{0}-b_{2}=2(n-1)\left(a_{1}+n a_{0}\right), \\
& b_{0}-b_{1}=n a_{1}+2(n-1) a_{0} .
\end{aligned}
$$

Åquivalent zu Satz 3.2 sind gewisse Abschätzungen für $F$ bzw. seine Bestandteile. Solche Abschätzungen wurden frühzeitig von P. GüNTHER [6, 7] angewendet.

Bei lorentzschen Metriken $g$ kann man Definitheitsschlüsse anwenden, wenn $|S|^{2}$ und $|W e y l|^{2}$ definit oder wenigstens semidefinit in den nichtverschwindenden Komponenten von $S$ bzw. Weyl sind. Wir betrachten im folgenden dafür hinreichende Bedingungen. Es sei $n \geqq 4$, die Signatur sei $(+-\cdots-)$ und die Indexkonvention abweichend vom bisherigen

$$
\begin{aligned}
& \alpha, \beta, \mu, v, \ldots=0,1,2, \ldots, n-1 ; \\
& i, j, k, l, \ldots=1,2, \ldots, n-1 \\
& I, J, K, L, \ldots=2, \ldots, n-1
\end{aligned}
$$


Satz 3.3: Gestattet die Metrik einen hyperflächenorthogonalen (abgekürat: hfo.) Killingvektor, d. h. ein Vektorfeld $v=v^{\star} \partial_{a}$ mit

so gilt

$$
v_{[\mu} \nabla_{\mathrm{a}} v_{\beta]}=0, \quad \nabla_{(\mathrm{a}} v_{\beta)}=0,
$$

$$
\begin{aligned}
& v_{[\mu} S_{\alpha] \beta} v^{\beta}=0, \quad v_{[2} C_{\alpha \beta] \mu \nu} v^{v}=0 \\
& |v|^{2}>0 \Rightarrow|S|^{2}, \quad \mid \text { Weyl }\left.\right|^{2} \text { positiv definit; } \\
& |v|^{8}=0 \Rightarrow|S|^{2}, \quad \mid \text { Weyl| }\left.\right|^{2} \text { pasitiv semidefinit } .
\end{aligned}
$$

Beweis: Aus (3.5) folgen lokale Darstellungen

mit

$$
v_{\alpha}=e^{2 \lambda} \partial_{\alpha} \varphi, \quad \nabla_{\alpha} v_{\beta}=2 \lambda_{[\alpha} v_{\beta]}, \quad \nabla_{\mu} \nabla_{a} v_{\beta}=2 \lambda_{\mu[a} v_{\beta]}
$$

$$
\lambda_{\alpha}:=\nabla_{\alpha} \lambda, \quad \lambda_{\alpha \beta}:=e^{-\lambda} \nabla_{\alpha} \nabla_{\beta} e^{\lambda}=\lambda_{\alpha} \lambda_{\beta}+\nabla_{\alpha} \lambda_{\beta}
$$

Im Falle $|v|^{2}>0$ kann man nach einem Argument von A. TradTMAN [19] $e^{2 \alpha}=|v|^{2}$ wählen und erhält dann:

$$
0=2 v^{\alpha} v^{\beta} \nabla_{\alpha} v_{\beta}=v^{\alpha} \nabla_{\alpha}|v|^{2} \Rightarrow v^{\alpha} \lambda_{\alpha}=0 .
$$

Im Falle $v^{2}=0$ erhält man einfacher:

In beiden Fällen erhält man weiter

$$
0=\nabla_{\alpha}|v|^{2}=2 v^{\beta} \nabla_{\alpha} v_{\beta}=-v^{\beta} \lambda_{\beta} v_{\alpha} \Rightarrow v^{\alpha} \lambda_{\alpha}=0
$$

$$
\lambda_{\alpha \beta} v^{\beta}=\lambda_{\beta} \lambda^{\beta} v_{\alpha}, \quad 0={\underset{v}{\mathfrak{E}}}_{\boldsymbol{E}} \Gamma_{\alpha \beta \mu}=\nabla_{\beta} \nabla_{\alpha} v_{\mu}+R_{\mu \alpha \beta} v^{\circ}
$$

und daraus

$$
R_{\alpha \beta \mu} v^{\nu}=2 v_{[\alpha} \lambda_{\beta]_{\mu}}, \quad R_{\alpha \beta} v^{\beta}=v_{\alpha} \cdot \Delta \lambda .
$$

Die Behauptung (3.6) ergibt sich hieraus durch Einsetzen der Definitionsformeln von $S$ und $W e y l$.

Im Falle $|v|^{2}>0$ normieren wir $v$ und wählen Koordinaten so, daß in einem bestimmten festen Punkt gilt

$$
v=\partial_{0}, \quad g=\left(d x^{0}\right)^{2}-d x^{i} d x^{i} .
$$

Die Bedingungen (3.6) bedeuten dann in dem festen Punkt

$$
S_{0 i}=0, \quad C_{0 i j k}=0,
$$

woraus sich ergibt

$$
|S|^{2}=S_{00}^{2}+S_{i j} S_{i j}, \quad|W e y l|^{2}=4 C_{0 i 0 j} C_{0 i 0 j}+C_{i j k l} C_{i j k l} .
$$

Offenbar sind hier $|S|^{2},|W e y l|^{2}$ positiv definit.

Im Falle $|v|^{2}=0$ wählen wir Koordinaten so, $\mathrm{da} \beta$ in einem bestimmten festen Punkt gilt

$$
v=\partial_{0}, . \quad g=2 d x^{0} d x^{1}-d x^{I} d x^{I} .
$$

Die Bedingungen (3.6) bedeuten dann in dem festen Punkt

$$
\begin{aligned}
& S_{00}=0, \quad S_{0 I}=0, \\
& C_{010 I}=0, \quad C_{01 I J} \equiv C_{0 I 1 J}-C_{1 I 0 J}=0, \quad C_{0 I O J}=0, \quad C_{0 I J K}=0,
\end{aligned}
$$


woraus sich ergibt

$$
\begin{aligned}
& |S|^{2}=2 S_{01}^{2}+S_{I J} S_{I J}, \\
& |W e y l|^{2}=4 C_{0101}^{2}+8 C_{0 I 1 J} C_{0 I 1 J}+C_{I J K L} C_{I J K L} .
\end{aligned}
$$

Offenbar sind hier $|S|^{2}$, $|W e y l|^{2}$ positiv semidefinit und es gilt in dem festen Punkt

$$
\begin{aligned}
|S|^{2}=0 \Leftrightarrow S=S_{11}\left(d x^{1}\right)^{2} & +2 S_{1 I} d x^{1} d x^{I} \\
|W e y l|^{2}=0 \Leftrightarrow W e y l= & 8 C_{011 I} d x^{0} \wedge d x^{1} d x^{2} \wedge d x^{I} \\
& +4 C_{1 I J J} d x^{1} \wedge d x^{I} d x^{1} \wedge d x^{J} \\
& +4 C_{1 I J K} d x^{1} \wedge d x^{I} d x^{J} \wedge d x^{K}
\end{aligned}
$$

Die zuletzt im Beweis behandelten Bedingungen an $S$, Weyl kann man auch koordinateninvariant formulieren: Aus (3.6), $|v|^{2}=0,|S|^{2}=0$ und $|W e y l|^{2}=0$ folgt:

$$
\begin{aligned}
& v_{[\mu} S_{\alpha][\beta} v_{v]}=0, \quad S_{\alpha \beta} v^{\beta}=0 ; \\
& C_{\alpha \beta \mu} v^{\beta} v^{\nu}=0, \quad v_{[\lambda} C_{\alpha \beta] \mu \nu} v^{\nu}=0, \quad v_{[\lambda} C_{\alpha \beta][\mu \nu} v_{\rho]}=0 ; \\
& v_{[\lambda} C_{\alpha] \beta \mu \nu} v^{\beta}=0 .
\end{aligned}
$$

Bei $n=4$ werden hierdurch wohlbekannte Typen von Tensoren charakterisiert: (3.9) charakterisiert einen Tensor $S$ vom Plebanskityp $[4 N]_{3} ;(3.10)$ charakterisiert einen Tensor Weyl vom Beltyp 3a; (3.11) charakterisiert einen Tensor Weyl vom Petrovtyp III (siehe dazu etwa $[10,1,12])$. Mathematisch gesehen handelt es sich um eine (nicht extreme) Ausartung der Tensoren; physikalisch gesehen handelt es sich um (nicht extreme) Strahlungsbedingungen.

Im Beweis von Satz 3.3 können $S$, Weyl sinngemäß durch Ric, Riem ersetzt werden: Gestattet die Metrik einen hfo. Killingvektor $v$, so gilt

$$
\begin{aligned}
& v_{[\mu} R_{\alpha] \beta} v^{\beta}=0, \quad v_{[\alpha} R_{\alpha \beta] \mu \mathrm{r}} v^{\prime}=0 ; \\
& |v|^{2}>0 \Rightarrow \mid \text { Ric }\left.\right|^{2}, \mid \text { Riem }\left.\right|^{2} \text { positiv definit; } \\
& |v|^{2}=0 \Rightarrow \mid \text { Ric }\left.\right|^{2}, \mid \text { Riem }\left.\right|^{2} \text { positiv semidefinit. }
\end{aligned}
$$

Aus (3.12), $|v|^{2}=0,|R i c|^{2}=0$ und $\mid$ Riem $\left.\right|^{2}=0$ folgt dann:

$$
\begin{aligned}
& v_{[\mu} R_{\alpha][\beta} v_{\nu]}=0, \quad R_{\alpha \beta} v^{\beta}=0 ; \\
& R_{\alpha \beta \mu v} v^{\beta} v^{v}=0, \quad v_{[\lambda} R_{\alpha \beta] \mu \nu} v^{\prime}=0, \quad v_{[\lambda} R_{\alpha \beta][\mu \nu} v_{\rho]}=0 \\
& v_{[\lambda} R_{\alpha \beta] \beta \mu \nu} v^{\beta}=0 .
\end{aligned}
$$

Nachdem wir die Krümmungsgrößen bei Existenz eines hfo. Killingvektors und bei etwaigen Zusatzbedingungen untersucht haben, bestimmen wir Normalformen für die lorentzsche Metrik $g$ selbst. Die der allgemeinen Relativitätstheorie entstammende Definition:

$$
g \text { statisch } \Leftrightarrow g \text { gestattet zeitartigen hfo. Killingvektor, }
$$

wurde in [16, 17] ergänzt durch die neue Begriffsbildung:

$g$ retardiert $\Leftrightarrow g$ gestattet lichtartigen hfo. Killingvektor.

Satz 3.4: Eine statische Metrik lät sich durch geeignete Koordinatenwahl auf die Form bringen

$$
g=g_{00}\left(x^{k}\right)\left(d x^{0}\right)^{2}+g_{i j}\left(x^{k}\right) d x^{i} d x^{i} .
$$

Eine statische Metrik mit $\mid$ Riem $\left.\right|^{2}=0$ ist flach. 
Eine retardierte Metrik läßt sich durch geeignete Koordinatenwahl auf die Form bringen

$$
g=2 g_{01}\left(x^{k}\right) d x^{0} d x^{1}+g_{i j}\left(x^{k}\right) d x^{i} d x^{j} \text {. }
$$

Eine retardierte Metrik mit $\mid$ Riem $\left.\right|^{2}=0$ ist eine $p p-W e l l e n-M e t r i k(:=$ plane-fronted wave metric with parallel rays) vom Kundtschen Typ, d. h. läkt sich durch geeignete Koordinatenwahl auf die Form bringen

mit

$$
g=, 2 d x^{0} d x^{1}+g_{11}\left(d x^{1}\right)^{2}+2 g_{1 I} d x^{1} d x^{I}-d x^{I} d x^{I}
$$

$$
\partial_{0} g_{11}=\partial_{0} g_{1 I}=0
$$

Eine retardierte Metrik mit

$$
R_{\alpha \beta \mu} R_{.}^{\alpha \mu \mu}=0
$$

ist eine-pp-Wellen-Metrik vom Robinsonschen Typ, d.h. läßt sich durch geeignete Koordinatenwahl auf die Form bringen

$$
g=2 d x^{0} d x^{1}+g_{11}\left(x^{1}, x^{K}\right)\left(d x^{1}\right)^{2}-d x^{I} d x^{I} .
$$

Das Vektorfeld $v=v^{a} \partial_{\alpha}=\partial_{0}$ ist bei (3.18), (3.19) ein hfo. Killingvektor und bei (3.20), (3.22) sogar ein kovariant konstanter Vektor.

Der Bew eis kann leicht mit den in $[15,16]$ dargelegten Methoden erbracht werden. Die obige zu (3.12) bis (3.17) gehörige Diskussion ist dabei von Nutzen

Neben den Spezialisierungen in den beiden vorstehenden Sätzen betrachten wir jetzt noch eine andere Art der Spezialișierung: Die Metrik $g$ oder genauer die riemannsche Mannigfaltigkeit $(M, g)$ heißt $\left(n^{\prime}, n^{\prime \prime}\right)$-zerlegbar, falls sich $M$ durch Koordinatensysteme überdecken läßt, in denen lokal gilt

$$
g=g^{\prime}+g^{\prime \prime}=g_{a b}\left(x^{c}\right) d x^{a} d x^{b}+g_{i j}\left(x^{k}\right) d x^{i} d x^{j},
$$

mit den Teildimensionen

$$
n^{\prime}=\operatorname{dim} g^{\prime} \geqq 1, \quad n^{\prime \prime}=\operatorname{dim} g^{\prime \prime} \geqq 1, \quad n^{\prime}+n^{\prime \prime}=n
$$

und der Indexkonvention

$$
a, b, c, \ldots=1,2, \ldots, n^{\prime} ; \quad i, j, k, \ldots=n^{\prime}+1, n^{\prime}+2, \ldots, n .
$$

Es erweist sich als zweckmäßig, die Schreibweise (3.23), (3.24) formal auch auf die Fälle „uneigentlicher Zerlegbarkeit"

$$
\begin{aligned}
& g^{\prime}=0, \quad g^{\prime \prime}=g, \quad n^{\prime}=0, \quad n^{\prime \prime}=n ; \\
& g^{\prime}=g, \quad g^{\prime \prime}=0, \quad n^{\prime}=n, \quad n^{\prime \prime}=0
\end{aligned}
$$

auszudehnen.

Satz 3.5: Eine Invariante $F=F[g]$ der Gestalt (3.1) genügt einer ,Additionsformel"

$$
g=g^{\prime}+g^{\prime \prime} \Rightarrow F[g]=F\left[g^{\prime}\right]+F\left[g^{\prime \prime}\right]+2 a_{0} R^{\prime} R^{\prime \prime}
$$

Ist hier g" flach, so gilt

$$
F[g]=F\left[g^{\prime}\right]=a_{2} \mid \text { Riem }\left.^{\prime}\right|^{2}+a_{1} \mid \text { Ric }\left.^{\prime}\right|^{2}+a_{0} R^{\prime 2},
$$


wobei die Größen

$$
\begin{aligned}
& \text { Riem } m^{\prime}=R_{a b c d} d x^{a} \wedge d x^{b} d x^{c} \wedge d x^{d}, \\
& R i c^{\prime}=R_{a b} d x^{a} d x^{b}, \quad R^{\prime}=R
\end{aligned}
$$

und die Operation $|. .|^{2}$ sowohl bezüglich $g^{\prime}$ als auch bezüglich g gedeutet werden können. Ist überdies $g^{\prime}$ definit und

$$
\begin{aligned}
& b_{2}^{\prime}:=4 a_{2}, \quad b_{1}^{\prime}:=4 a_{2}+\left(n^{\prime}-2\right) a_{1}, \\
& b_{0}^{\prime}:=4 a_{2}+2\left(n^{\prime}-1\right)\left(a_{1}+n^{\prime} a_{0}\right) .
\end{aligned}
$$

gesetzt, so folgt aus jeder der Bedingungen

$$
\begin{array}{lll}
n^{\prime} \geqq 4 & \text { und } & \operatorname{sgn} b_{2}{ }^{\prime}=\operatorname{sgn} b_{1}{ }^{\prime}=\operatorname{sgn} b_{0}{ }^{\prime} \neq 0, \\
n^{\prime}=3 & \text { und } & \operatorname{sgn} b_{1}^{\prime}=\operatorname{sgn} b_{0}{ }^{\prime} \neq 0, \\
n^{\prime}=2 & \text { und } & b_{0}{ }^{\prime} \neq 0
\end{array}
$$

die Definitheit von $F$ im Krümmungstensor.

\section{§4. Berechnung von Spuren}

Motiviert durch die Formeln (2.2) und (2.4) betrachten wir lineare Operatoren von besonderer Gestalt.

Definition: Es sei $A^{(\natural)}$ ein gemäß

$$
\left(A^{(a)} u\right)_{\alpha_{1} \alpha_{1} \ldots \alpha_{a}}=A_{\alpha_{2} \alpha_{2} \ldots \alpha_{q}}^{\mu_{1} \mu_{2} \ldots \mu_{q}} u_{\mu_{1} \mu_{3} \ldots \mu_{e}}
$$

auf kovariante $q$-Tensorfelder $u$ wirkender linearer Operator. Der gemä $B$

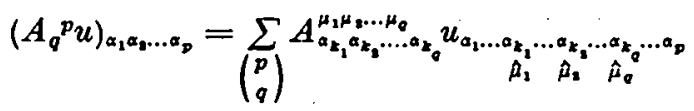

auf kovariante $p$-Tensorfelder $u(p \geqq q)$ wirkende lineare Operator $A_{q}^{p}$ heiße $p$-Erweiterung von $A^{(q)}$. In (4.2) erfolge die Summation über alle $\left(\begin{array}{l}p \\ q\end{array}\right)$ Stück $q$-stel-
ligen Teilfolgen $\left(k_{1}, k_{2}, \ldots, k_{q}\right)$ der Folge $(1,2, \ldots, p)$. Für $q=1$ bzw. $q=2$ spezialisiert sich (4.2) zu

$$
\begin{aligned}
& \left(A_{1}{ }^{p} u\right)_{\alpha_{1} \alpha_{2} \ldots \alpha_{p}}=\sum_{k=1}^{p} A_{\alpha_{k}}^{u} u_{\alpha_{2} \ldots \alpha_{k} \ldots \alpha_{p}} \quad(p \geqq 1), \\
& \left(A_{2}^{p} u\right)_{\alpha_{1} \alpha_{2} \ldots \alpha_{\nu}}=\sum_{k=1}^{p-1} \sum_{l=k+1}^{p} A_{\alpha_{k} \alpha_{l}}^{\mu \nu} u_{\alpha_{1} \ldots \alpha_{k} \ldots \alpha_{l} \ldots \alpha_{\nu}} \quad(p \geqq 2) .
\end{aligned}
$$

Der Autor hat in [14] folgendes bewiesen:

Satz 4.1: Ändern sich die Komponenten $A_{a_{1} \alpha_{2} \ldots \alpha_{q}}^{\mu_{1} \mu_{1} \ldots \mu_{q}}$ von $A^{(q)}$ bei gleichzeitiger Vertauschung von $\mu_{k}$ mit $\mu_{l}$ und von $\alpha_{k}$ mit $\alpha_{l}(1 \leqq k, l \leqq q)$ nicht, so ist die p-Erweiterung $A_{q}{ }^{p}$ von $A^{(q)}$ mit der Alternierung bzw. Mischung (d.h. mit der natürlichen Projektion auf $\Lambda^{p} T^{*}$ bzw. $\Pi^{p} T^{*}$ ) vertauschbar.

Nunmehr lassen sich die Formeln (2.2), (2.4) nachträglich rechtfertigen:

Satz 4.2: Die Anwendung der operatorwertigen 2-Form $K_{\alpha \beta} d x^{\alpha} \wedge d x^{\beta}$ gemä $\beta$ (2.2) ist mit der natürlichen Projektion auf $A^{p} T^{*}$ bzw. auf $\Pi^{p} T^{*}$ bzw. auf $\Pi^{p} T^{*} / g$ vertausch- 
bar. Die Anwendung des Operators $Z$ gemä $\beta$ (2.4) ist mit der natürlichen Projektion auf $\Lambda^{p} T^{*}$ bzw. auf $\Pi^{p} T^{*}$ vertauschbar.

Beweis: Sinngemäß ist $K_{\alpha \beta}$ vom Typ $A_{1}{ }^{p}$ und deshalb mit der Alternierung bzw. Mischung vertauschbar. Ist $u$ eine spurfreie symmetrische Form, so ist $K_{a \beta} u$ auf Grund von $R_{\alpha \beta \mu}{ }^{\mu} .=0$ wieder spurfrei. In der Zerlegung gemäß (2.4)

$$
Z=Z_{1}^{p}+Z_{2}^{p} \text { mit Komponenten } Z_{a}^{\mu}=R_{\alpha}{ }^{\mu}, \quad Z_{a \beta}^{\mu \nu}=-2 R^{\mu}{ }^{\mu}{ }_{\alpha \beta} \text {. }
$$

von Operatoren $Z^{(1)}, Z^{(2)}$ sind auf Grund von $Z_{\alpha \beta}^{\mu \nu}=Z_{\beta \alpha}^{\mu \mu}$ beide Summanden $Z_{1}{ }^{p}, Z_{2}{ }^{p}$ mit der Alternierung bzw. Mischung vertauschbar.

Als $S$ pur eines Operators $A^{(p)}$ bezüglich $g$ und $E$ hat man

$$
\operatorname{Tr} A^{(p)}:=P_{\beta_{1} \beta_{2} \ldots \beta_{p}}^{\alpha_{1} \alpha_{2} \ldots \alpha_{p}} A_{a_{1} \alpha_{1} \ldots \alpha_{p}}^{\beta_{1} \beta_{2} \ldots \beta_{p}}
$$

zu definieren. Speziell gilt

$$
\operatorname{Tr} I=\operatorname{Tr} P^{(p)}=P_{\alpha_{1} a_{1} \ldots \alpha_{p}}^{a_{1} a_{1} \ldots \alpha_{y}}=N_{0}^{p}
$$

Die Definition (4.6) läßt sich etwa so motivieren: Die Fasern von $E^{p}$ (aufgefaßt als Unterbündel von $\otimes p T^{*}$ ) werden jeweils von den Elementen

$$
e^{\alpha_{2} \alpha_{2} \ldots \alpha_{p}}:=P_{\beta_{2} \beta_{2} \ldots \beta_{p}}^{\alpha_{1} \alpha_{1} \ldots \alpha_{p}} d x^{\beta_{2}} \otimes \cdots \otimes d x^{\beta_{p}}
$$

aufgespannt und die Fasern des zugehörigen dualen Bündels $E^{p *}$ (aufgefaßt als Unterbündel von $\otimes{ }^{p} T$ ) jeweils von den

$$
e_{\beta_{1} \beta_{2} \ldots \beta_{p}}:=P_{\beta_{1} \beta_{1} \ldots \beta_{p}}^{a_{1} \alpha_{2} \ldots \alpha_{p}} \partial_{a_{1}} \otimes \cdots \otimes \partial_{\alpha_{p}} \text {. }
$$

Es gelten die „,Dualitätsrelation“

$$
\left\langle e_{\beta_{2} \beta_{2} \ldots \beta_{p}}, e_{a_{1} \alpha_{2} \ldots \alpha_{p}}\right\rangle=P_{\beta_{1} \beta_{2} \ldots \beta_{p}}^{\alpha_{1} \alpha_{2} \ldots \alpha_{p}}
$$

und (4.6) in einer der gewohnten Spurdefinition nachgebildeten Form

$$
\operatorname{Tr} A^{(p)}=\sum_{a_{1}, \alpha_{1}, \ldots, \alpha_{p}}\left\langle e_{\alpha_{1} \alpha_{2} \ldots \alpha_{p}}, A^{(p)} e^{\alpha_{1} \alpha_{2} \ldots \alpha_{p}}\right\rangle
$$

Wir berechnen die Spur von $p$-Erweiterungen bzw. gewisser Produkte von $p$-Erweiterungen.

Satz 4.3: Es gilt in den vereinbarten Bezeichnungen

$$
\begin{aligned}
& \operatorname{Tr} A_{q}^{p}=N_{q}^{p} \operatorname{Tr} A^{(q)}, \\
& \operatorname{Tr}\left(A_{1}^{p} B_{1}^{p}\right)=N_{1}^{p} A_{\beta}^{a} B_{a}^{\beta}+2 N_{2}^{p} P_{\beta_{1} \beta}^{\alpha_{1} \alpha_{3}} A_{\alpha_{1}}^{\beta_{1}} B_{\alpha_{q}}^{\beta_{2}} .
\end{aligned}
$$

Unter der Voraussetzung $A_{\alpha \beta}^{\mu r}=A_{\beta \alpha}^{\nu \mu}, B_{\alpha \beta}^{\mu \nu}=B_{\beta a}^{\nu \mu}$ gilt weiter

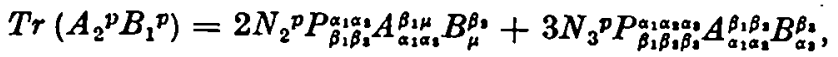

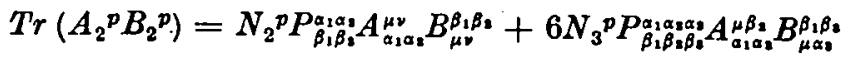

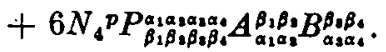

Beweis: Die Verjüngung $\beta_{1}=\alpha_{1}, \beta_{2}=\alpha_{2}, \ldots, \beta_{p}=\alpha_{p}$ von

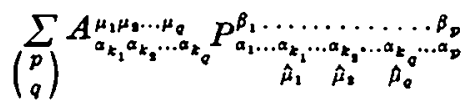


ergibt unter Beachtung von (1.10)

$$
\sum_{\left(\begin{array}{l}
p \\
q
\end{array}\right)} A_{\alpha_{k_{1} \alpha_{k_{1}} \ldots \alpha_{k_{q}}}}^{\mu_{1} \mu_{2} \ldots \mu_{q}} \frac{N_{0}^{p}}{N_{0}^{q}} P_{\mu_{1} \mu_{2} \ldots \mu_{q}}^{\alpha_{k_{1}} \alpha_{k_{1}} \ldots \alpha_{k_{q}}}=N_{q}^{p} \operatorname{Tr} A^{(q)} .
$$

Die Komponenten von $A_{1}^{p} B_{1}{ }^{p} u$ sind gleich

$$
\sum_{l} A_{\alpha_{l}}^{\nu}\left(\sum_{k<l} B_{\alpha_{k}}^{\mu} u_{\alpha_{1} \ldots \alpha_{k} \ldots a_{l} \ldots \alpha_{p}}+B_{\hat{\imath}}^{\mu} u_{\alpha_{1} \ldots \alpha_{l} \ldots \alpha_{p}}+\sum_{k>l} B_{\alpha_{k}}^{\mu} u_{\alpha_{1} \ldots \alpha_{l} \ldots \alpha_{k} \ldots \alpha_{p}}\right) \cdot
$$

Daraus erhalten wir

$$
\begin{aligned}
A_{1}{ }^{p} B_{1}{ }^{p}= & p \text {-Erweiterung von } A^{(1)} B^{(1)} \\
& +p \text {-Erweiterung von }\left(A^{(1)} \otimes B^{(1)}+B^{(1)} \otimes A^{(1)}\right)
\end{aligned}
$$

und daraus (4.9) unter Beachtung von (4.8). Die Formeln (4.10), (4.11) können ebenfalls durch direkte Ausrechnung der Komponenten bewiesen werden. $\mathrm{Da}$ aber die Ausdrücke sehr umfangreich werden, unterdrücken wir diese Rechnungen

Im folgenden spielt die Größe

$$
M_{1}{ }^{p}:=\left(N_{1}{ }^{p}+\varepsilon N_{2}{ }^{p}\right) / N_{0}{ }^{p}=\frac{p}{n}+\frac{\varepsilon}{N_{0}{ }^{2}}\left(\begin{array}{l}
p \\
2
\end{array}\right)
$$

eine gewisse Rolle. Für $E=\Lambda T^{*}$ bzw. $=\Pi T^{*}$ erhalten wir aus (1.4) mit $r=1$

$$
M_{1}^{p}=N_{2}^{p+1} / N_{0}^{p}
$$

und ergänzen dies durch die Definition einer weiteren Größe

$$
M_{2}^{p}:=N_{4}^{p+2} / N_{0}^{p} \text {. }
$$

Satz 4.4: Es gilt die Spurformel.

$$
\operatorname{Tr} K_{\alpha \beta} K^{\alpha \beta}=-N_{0}^{p} \cdot M_{1}^{p} \cdot \mid \text { Riem }\left.\right|^{2} .
$$

Für $E=A T^{*} b z w .=\Pi T^{*}$ gelten weiter die Spurformeln

$$
\begin{aligned}
& \operatorname{Tr} Z=N_{2}^{p+1} \cdot R, \\
& \operatorname{Tr} Z^{2}=N_{2}{ }^{p+1} \cdot|R i c|^{2}+N_{4}^{p+2} \cdot\left[(2+\varepsilon)|R i e m|^{2}+4 \varepsilon|R i c|^{2}+R^{2}\right] .
\end{aligned}
$$

Beweis: Man wendet Satz 4.3 sinngemä $B$ auf $K_{\alpha \beta} K^{\alpha \beta}$ und auf die Bestandteile von

$$
Z=Z_{1}{ }^{p}+Z_{2}{ }^{p}, \quad Z^{2}=\left(Z_{1}{ }^{p}\right)^{2}+Z_{1}{ }^{p} Z_{2}{ }^{p}+Z_{2}{ }^{p} Z_{1}{ }^{p}+\left(Z_{2}{ }^{p}\right)^{2}
$$

an. Satz 1.1 erweist sich in der Version

$$
2 P_{\beta_{1} \beta_{2}}^{\alpha_{1} \alpha_{1}} A_{\alpha_{2}}^{\beta_{3}}= \begin{cases}\varepsilon A_{\beta_{1}}^{\alpha_{1}} & \text { für } A_{\alpha \beta}=A_{[\alpha \beta]}, \\ \delta_{\beta_{1}}^{\alpha_{1}} A_{\mu}^{\mu}+\gamma A_{\beta_{2}}^{\alpha_{1}} & \text { für } A_{\alpha \beta}+A_{(\alpha \beta)}\end{cases}
$$

als nützlich. Für $E=\Lambda T^{*}$ bzw. $=\Pi T^{*}$ werden die Komponenten von $P^{(3)}$ mittels der Entwicklungsformel (1.12) auf die von $P^{(2)}$. zurückgeführt und die Komponenten von $P^{(4)}$ werden analog auf die von $P^{(3)}$ zurückgeführt. Die Formel (1.4) kommt mit $r=1$ und mit $r=2$ zur Anwendung

Die bisherigen Vorbereitungen zielen vor allem darauf, die Spur des Koinzidenzwertes des 2 . Hadamardkoeffizienten zu berechnen. 
Satz 4.5: Die Größe $180 \operatorname{Tr} U_{2} / N_{0}^{p}$ ist beziehentlich gleich dem Produkt des Zeilenvektors

$\left(1-15 M_{1}^{p},-1, \frac{5}{2}, 6\right)$ für $\Delta$,
$\left(1-15 M_{1}^{p},-1, \frac{5}{S}\left(\frac{n-4}{n-1}\right)^{2},-\frac{3}{2}\left(\frac{n-6}{n-1}\right)\right)$ für $\Delta-\frac{n-2}{4(n-1)} R I$,

$$
\left(1, M_{1}^{p}, M_{2}^{p}\right) \cdot\left(\begin{array}{llll}
1 & -1 & \frac{5}{2} & 6 \\
-15 & 90 & 30 \varepsilon & 30 \varepsilon \\
90(2+\varepsilon) & 360 \varepsilon & 90 & 0
\end{array}\right) \text { für } \Delta+\varepsilon Z
$$

mit dem Spaltenvektor

$$
\left(\begin{array}{l}
\mid \text { Riem }\left.\right|^{2} \\
\mid \text { Ric }\left.\right|^{2} \\
R^{2} \\
\Delta R
\end{array}\right)
$$

Zum Beweis ist $C=0,-\frac{n-2}{4(n-1)} R I, \varepsilon Z$ in

$$
\begin{aligned}
180 U_{2} \doteq & \left(\mid \text { Riem }\left.\right|^{2}-|R i c|^{2}+\frac{5}{2} R^{2}+6 \Delta R\right) I \\
& +15 K_{\alpha \beta} K^{\alpha \beta}+90 C^{2}+30 R C+30 \Delta C
\end{aligned}
$$

einzusetzen und die Spur unter Benutzung von (4.7), (4.15), (4.16) zu berechnen. Für den Fall $E=\Lambda T^{*}, L=\Delta-Z=-(d \delta+\delta d)$ reproduziert unser Ergebnis (4.18) Formeln aus der Literatur $[8,13]$

Wir untersuchen die Größe $M_{1}{ }^{p}$ etwas genauer:

Satz 4.6: In der Reihenfolge

$$
E=A T^{*}, \otimes T^{*}, \Pi T^{*}, \Pi T^{*} / g
$$

gilt beziehentlich

$$
M_{1}^{p}=\frac{p(n-p)}{n(n-1)}, \frac{p}{n}, \frac{p(n+p)}{n(n+1)}, \frac{p(n+p-2)}{n(n-1)} .
$$

Eine Ungleichung bzw. Gleichung (man lese jeweils eins der Zeichen $>,=,<, \geqq$; !)

$$
A \cdot M_{1}^{p} \gtreqless B
$$

ist beziehentlich äquivalent zu einer Ungleichung bzw. Gleichung

$$
\begin{array}{ll}
A\left(p-\frac{n}{2}\right)^{2} \lessgtr A \frac{n^{2}}{4}-B n(n-1) & \text { für } \Lambda T^{*}, \\
A p \geqq B n & \text { für } \otimes T^{*}, \\
A\left(p+\frac{n}{2}\right)^{2} \gtreqless A \frac{n^{2}}{4}+B n(n+1) & \text { für } \Pi T^{*}, \\
A\left(p+\frac{n}{2}-1\right)^{2} \gtreqless A\left(\frac{n}{2}-1\right)^{2}+B n(n-1) & \text { für } \Pi T^{*} / g .
\end{array}
$$


Die Größe $M_{1}^{p}=M_{1}^{p}(n, E)$ hängt monoton

- fallend von $n a b$,

- steigend von $p a b$,

- steigend von $E$ bezüglich der Reihenfolge (4.19) ab.

Dabei sei für $E=\Lambda T^{*}$ noch $p \leqq \frac{n}{2}$ vereinbart.

Kraft der Dualität bei altermierenden Differentialformen ( $M$ sei orientierbar!) bedeutet die letztgenannte Vereinbarung keine Einschränkung der Allgemeinheit.

Das folgende Ergebnis wird für $§ 6$ bereitgestellt:

Satz 4.7: Für $n \geqq 3, E^{p} \neq E^{0}$ und $\neq \Lambda^{\frac{n}{2}} T^{*}$ gill:

$$
K_{\alpha \beta} K^{\alpha \beta}=0 \Rightarrow R_{\alpha \beta \mu} R_{\cdots \lambda}^{\alpha \beta \mu}=0 .
$$

Beweis: Die Komponenten von $\boldsymbol{K}_{\alpha \beta} K^{\alpha \beta}$ sind gleich

$$
\sum_{k} L_{\alpha_{k}}^{\mu} P_{\alpha_{1} \ldots \alpha_{k} \ldots \alpha_{p}}^{\beta_{1} \ldots \beta_{p}}+2 \sum_{k<l} L_{\alpha_{k} \alpha_{t}}^{\mu v} P_{a_{1} \ldots \ldots \alpha_{\hat{\mu}} \ldots \ldots \beta_{j} \ldots \alpha_{p}}^{\beta_{1} \ldots \alpha_{p}}
$$

mit den Abkürzungen

$$
L_{\alpha_{1 \alpha} \alpha_{z}}^{\beta_{1} \beta_{2}}:=R_{\mu v x_{2}}^{\beta_{1}}: R_{. .}^{\mu \nu}, \quad L_{\alpha}^{\beta}:=L_{\alpha \mu}^{\mu \beta} .
$$

Aus (4.15) folgt:

$$
\operatorname{Tr} K_{\alpha \beta} K^{\alpha \beta}=0 \Rightarrow L_{\alpha}{ }^{\alpha}=0 .
$$

Unter Beachtung dessen wird die $(p-1)$-fache Verjüngung $\beta_{2}=\alpha_{2}, \ldots, \beta_{p}=\alpha_{p}$ von (4.24) ausgerechnet: Sie ist gleich $L_{\alpha_{1}}^{\beta_{1}}$ multipliziert beziehentlich mit

$$
\begin{aligned}
& N_{1}^{p}+2 \frac{n+1}{n} N_{2}^{p}+2 \frac{n+4}{n+2} N_{3}^{p} \text { für } \Pi T^{*} / g \\
& N_{1}^{p}+3 \varepsilon N_{2}^{p}+2 \varepsilon^{2} N_{3}^{p} \text { sonst. }
\end{aligned}
$$

Dieser Faktor ist offenbar positiv außer eventuell für $A T^{*}, 2 \leqq p \leqq n-1$. Im letzteren Falle ist der Faktor

$$
\frac{n-2 p}{p-1}\left(\begin{array}{l}
n-3 \\
p-2
\end{array}\right) \neq 0 \text { für } p \neq \frac{n}{2} \text {. }
$$

\section{§ 5. Ergebnisse zur spektralen Geometrie}

In diesem Abschnitt setzen wir durchweg die Mannigfaltigkeit $M$ als geschlossen und die Metrik $g$ als definit voraus. Bei Integration von $\operatorname{Tr} U_{2}$ über $M$ verschwindet jeweils der Term mit $\Delta R$, und wir erhalten aus Satz 4.5 die Darstellung

$$
180 U_{2}(M) / N_{0}^{p}=a_{2}\|R i e m\|^{2}+a_{1}\|R i c\|^{2}+a_{0}\|R\|^{2},
$$


wobei der hierdurch erklürte Zeilenvektor $\left(a_{2}, a_{1}, a_{0}\right)$ beziehentlich gleich ist

$$
\begin{array}{ll}
\left(1-15 M_{1}^{p},-1, \frac{5}{2}\right) & \text { für } \Delta, \\
\left(1-15 M_{1}^{p},-1, \frac{5}{8}\left(\frac{n-4}{n-1}\right)^{2}\right) & \text { für } \Delta-\frac{n-2}{4(n-1)} R I, \\
\left(1, M_{1}{ }^{p}, M_{2}{ }^{p}\right) \cdot\left(\begin{array}{lll}
1 & -1 & \frac{5}{2} \\
-15 & 90 & 30 \varepsilon \\
90(2+\varepsilon) & 360 \varepsilon & 90
\end{array}\right) \text { für } \Delta+\varepsilon Z .
\end{array}
$$

Die sinngemäß angewandte Transformation aus Satz 3.1 liefert für die nichtkanonischen Laplacians (für den kanonischen Laplacian soll diese Transformation in einer späteren Arbeit abgehandelt werden):

$$
\left(\begin{array}{l}
b_{2} \\
b_{1} \\
b_{0}
\end{array}\right)=\left(\begin{array}{lll}
4 & 0 & 0 \\
4 & n-2 & 0 \\
4 & 2(n-1) & 2(n-1) n
\end{array}\right)\left(\begin{array}{l}
a_{2} \\
a_{1} \\
a_{0}
\end{array}\right)=\left(\begin{array}{r}
4-60 M_{1}{ }^{p} \\
6-n-60 M_{1}{ }^{p} \\
\cdot b_{0}{ }^{0}-60 M_{1}{ }^{p}
\end{array}\right)
$$

mit der Abkürzung

$$
b_{0}{ }^{0}:= \begin{cases}5 n^{2}-7 n+6 & \text { für } \Delta \\ \frac{n-6}{4(n-1)}\left(5 n^{2}-18 n+4\right) & \text { für } \Delta-\frac{n-2}{4(n-1)} R I .\end{cases}
$$

(Offenbar ist $b_{0}{ }^{0}$ der Wert von $b_{0}$ für $p=0$.) Aus den Formeln (5.3), (5.4) ergeben sich die folgenden Monotonieeigenschaften:

Satz 5.1: Für die nichtkanonischen Laplacians hängen die Größen $b_{2}, b_{1}, b_{0}$ monoton

- fallend von $p a b$,

- fallend von E bezüglich der Reihenfolge (4.19) $a b$,

- fallend von $L$ bezüglich der Reihenfolge $\Delta, \Delta-\frac{n-2}{4(n-1)} R I a b$.

Die Größen $b_{2}, b_{0}$ hängen monoton steigend von $n$ ab. Dabei sei jeweils für $E .=\Lambda T^{*}$ noch $p \leqq \frac{n}{2}$ vereinbart.

Beweis: Die Abhängigkeit von $p$ und von $E$ wird nur durch $M_{1}{ }^{p}$ vermittelt, und $b_{2}, b_{1}, b_{0}$ verhalten sich wie $\left(-M_{1}^{p}\right)$. Da $b_{2}, b_{1}$ für die beiden Laplacians übereinstimmen, sind die Ausdrücke $b_{0}$ zu vergleichen. Für $n \geqq 2$ gilt aber

$$
4(n-1)\left(5 n^{2}-7 n+6\right) \geqq(n-6)\left(5 n^{2}-18 n+4\right) .
$$

Die Monotonie von $b_{2}, b_{1}$ in $n$ folgt daraus, daß für $n \geqq 2$ die Ausdrücke

$$
\left(-M_{1}^{p}\right), \quad 5 n^{2}-7 n+6, \quad 5 n^{2}-18 n+4, \frac{n-6}{n-1}
$$

monoton steigend sind 
Nachdem unsere Vorbereitungen soweit gediehen sind, kommen wir zu zentralen Ergebnissen:

Theorem 5.1: Es sei eine der folgenden Bedingungen an $E, n, p$ erfüllt:

$$
\begin{aligned}
& n=2 ; \\
& 2 \leqq n \leqq 5 \text { und } p=0 ; \\
& 60 p>n\left(5 n^{2}-7 n+6\right) \text { für } \otimes T^{*}, \\
& 60\left(p+\frac{n}{2}\right)^{2}>n\left(5 n^{3}-2 n^{2}+14 n+6\right) \text { für } \Pi T^{*}, \\
& 60\left(p+\frac{n}{2}-1\right)^{2}>5 n^{4}-12 n^{3}+28 n^{2}-66 n+60 \text { für } \Pi T^{*} / g ; \\
& 12 p \geqq n^{3} \text { für } \otimes T^{*} ; \\
& \sqrt{12} p \geqq n^{2} \text { für } \Pi T^{*} \text { bzw. } \Pi T^{*} / g .
\end{aligned}
$$

(4) $\quad 12 p \geqq n^{3} \quad$ für $\otimes T^{*}$;

Wenn dann der reine Laplacian $\Delta$ isospektral zum Laplacian einer flachen Mannigfaltigkeit ist, so ist $(M, g)$ flach.

Beweis: Aus (3.4) und (5.2) erhalten wir

$$
b_{2}-b_{1}=n-2, \quad b_{0}-b_{2}=(n-1)(5 n-2) \Rightarrow b_{1} \leqq b_{2}<b_{0} .
$$

Wir schließen:

$$
\Rightarrow b_{0}=12-60 M_{1}^{p} \neq 0 \text { aus Teilbarkeitsgründen; }
$$

(2) $\quad \Rightarrow b_{1}=6-n>0$;

(3) $\quad \Rightarrow b_{0}<0$ unter Benutzung von Satz 4.6.

Die Abschätzung (4) ist gröber als die Abschätzung (3): Die Differenzen ,grobe Abschätzung (4) - feine Abschätzung (3)"

\begin{tabular}{|c|c|c|c|c|}
\hline$\underset{\downarrow}{E}=, \quad n=\rightarrow$ & 3 & 4 & 5 & 6 \\
\hline$\otimes T^{*}$ & 2 & 4 & 9 & 15 \\
\hline$\Pi T^{*}$ & 2 & 3 & $\mathbf{5}$ & 8 \\
\hline$\Pi T^{*} / g$ & 2 & 3 & 5 & 7 \\
\hline
\end{tabular}

$$
\begin{array}{ll}
7 n-6 & \text { für } \otimes T^{*}, \\
\alpha n^{2}+n-6 & \text { für } \Pi T^{*}, \\
(\alpha+10) n^{2}-(2 \alpha+9) n+6 & \text { für } \Pi T^{*} / g
\end{array}
$$

mit $\alpha:=2+10 \sqrt{3}$ sind für $n \geqq 2$ positiv, so daß $(4) \Rightarrow(3)$. Aus jeder der Bedingungen (1) bis (4) folgt also die Definitheit des Ausdrucks (5.1) und damit $U_{2}(M)=0$ $\Rightarrow$ Riem $=0$

Die „feinen Abschätzungen“ (3) kann man leicht auf konkrete Werte von $n$ und $p$ spezialisieren. Beispielsweise wird (3) erfüllt, wenn $p$ größer oder gleich der aus der Tabelle

entnommene Schranke ist. 
Theorem 5.2: Es sei eine der folgenden Bedingungen erfüllt:

$$
\begin{aligned}
& n=2 \text { oder }=3 ; \\
& 2 \leqq n \leqq 6 \text { und } p \geqq 1 ; \\
& n \geqq 7 \text { und } \\
& 240(n-1) p>n(n-6)\left(5 n^{2}-18 n+4\right)=: \varphi(n) \quad \text { für } \otimes T^{*}, \\
& 240(n-1)\left(p+\frac{n}{2}\right)^{2}>60 n^{2}(n-1)+(n+1) \varphi(n) \quad \text { für } \Pi T^{*}, \\
& 240\left(p+\frac{n}{2}-1\right)^{2}>60(n-2)^{2}+\varphi(n) \\
& 48 p \geqq n^{3} \quad \text { für } \otimes T^{*}, . \\
& \sqrt{48} p \geqq n^{2} \text { für } \Pi T^{*} \text { bzw. } \Pi T^{*} / g .
\end{aligned}
$$

(4) $\quad 48 p \geqq n^{3} \quad$ für $\otimes T^{*}$,

Wenn dann der Laplacian mit verschwindender Cottoninvariante $\Delta-\frac{n-2}{4(n-1)} R I$ isospektral zum Laplacian einer flachen Mannigfaltigkeit ist, so ist $(M, g)$ flach.

Beweis: Aus (3.4) und (5.2) erhalten wir

$$
\begin{aligned}
& b_{2}-b_{1}=n-2, \quad 4(n-1)\left(b_{0}-b_{2}\right)=5 n(n-4)^{2}-8(n-1)^{2}, \\
& 4(n-1)\left(b_{0}-b_{1}\right)=n(n-6)(5 n-14) .
\end{aligned}
$$

Es folgt

$$
b_{0} \leqq b_{1}<b_{2} \text { für } 3 \leqq n \leqq 6 \text { und } b_{1}<b_{2}<b_{0} \text { für } n \geqq 7 .
$$

Wir schließen

$$
\begin{aligned}
& n=2 \Rightarrow b_{0} \neq 0 \text { wie bei } \Delta ; \\
& n=3 \text { und } p=0 \Rightarrow 8 b_{0}=15>0 ; \\
& 2 \leqq n \leqq 6 \text { und } p=1 \Rightarrow n b_{2}=4 n-60<0 ; \\
& 2 \leqq n \leqq 6 \text { und } p \geqq 1 \Rightarrow b_{2}<0 \text { wegen Monotonie in } p \text { nach Satz 5.1; } \\
& (3) \Rightarrow b_{0}<0 \text { unter Benutzung von Satz 4.6. }
\end{aligned}
$$

Die Abschätzung (4) ist gröber als die Abschätzung (3): Die Differenzen ,,grobe Abschätzung (4) - feine Abschätzung (3)"

$$
\begin{array}{ll}
43 n^{2}-112 n+24 & \text { für } \otimes T^{*}, \\
(2 \alpha-5) n^{3}-(2 \alpha+8) n^{2}-44 n+12 & \text { für } \Pi T^{*}, \\
\alpha n^{2}-(2 \alpha+4) n+6 & \text { für } \Pi T^{*} / g
\end{array}
$$

mit $\alpha:=12+5 \sqrt{3}$ sind für $n \geqq 3$ positiv so daß $(4) \Rightarrow(3)$. Wie beim Beweis von Theorem 5.1 ergibt sich Riem $=0$

$\mathrm{Zu}$ den beiden Theoremen sind noch einige Bemerkungen angebracht:

1. Der ,,allgemeine Fall" bei den Beweisen ist $\operatorname{Max} b_{k}=b_{0}<0$. Ausnahmen davon liegen für spezielle (kleine) Werte von $n$ bzw. von $p$ vor. Im ,allgemeinen Fall" 
gilt nach Satz 5.1 das folgende Prinzip. Aussagen von der Art:

$L$ isospektral zum Laplacian eines flachen $(\tilde{M}, \tilde{g}) \Rightarrow(M, g)$ flach

sind um so leichter zu erhalten, je

- kleiner $n$ ist,

- größer $p$ ist,

- weiter rechts $E$ aus der Folge (4.19) gewählt wird.

Sie sind ferner für $\Delta-\frac{n-2}{4(n-1)} R I$ leichter als für $\Delta$ zu erhalten.

2. Die Punkte (1), (2) sind jeweils so gemeint, da $B E=\Lambda T^{*}$ mit erfaßt ist; bei (3), (4) ist dagegen $A T^{*}$ jeweils nicht mit erfaßt. Der Grund dafür ist: Für $E^{p}=\Lambda^{p} T$, $p \geqq 1$, folgt aus $n \geqq 3, L=\Delta$ bzw. aus $n \geqq 7, L=\Delta-\frac{n-2}{4(n-1)} R I$ :

$$
b_{1}<0<b_{0} \Rightarrow U_{2}(M) \text { indefinit. }
$$

3. Auf die ,groben Abschätzungen" (4) kommt man auf naheliegende Weise, wenn man nämlich $M_{1}{ }^{p}$ bzw. $b_{0}$ formal durch ihre asymptotischen Ausdrücke bei $n \rightarrow \infty$ und $\frac{p}{n} \rightarrow \infty$ ersetzt:

$$
\begin{array}{ll}
M_{1}{ }^{p} \approx\left(\frac{p}{n}\right)^{2} & \text { für } \Pi T^{*} \text { und für } \Pi T^{*} / g, \\
b_{0} \approx 5 n^{2}-60 M_{1}^{p} & \text { für } \Delta, \\
b_{0} \approx \frac{5}{4} n^{2}-60 M_{1}^{p} & \text { für } \Delta-\frac{n-2}{4(n-1)} R I .
\end{array}
$$

(Beachte: Für $\otimes T^{*}$ gilt $M_{1}{ }^{p}=\frac{p}{n}$ und für $A T^{*}$ hat $\frac{p}{n} \rightarrow \infty$ keinen Sinn.) Nachträglich erweisen sich dann die so gewonnenen Abschätzungen sogar als für alle $n \geqq 2$ brauchbar. Die Tabelle in der Einleitung ist nur eine modifizierte Form der Abschätzungen (4).

4. Für $n=6, p=0$ ergibt sich eine Nullstelle von $b_{1}$ bzw. von $b_{1}, b_{0}$ gleichzeitig:

$$
180 U_{2}(M)= \begin{cases}\|W e y l\|^{2}+\frac{12}{5}\|R\|^{2} & \text { für } L=\Delta, \\ \|W e y l\|^{2} & \text { für } L=\Delta-\frac{1 !}{5} R I .\end{cases}
$$

(Der allgemeinen Frage nach Nullstellen der $b_{k}$ soll in einer späteren Arbeit nachgegangen werden.)

Aus der letzten Bemerkung ergeben sich zwanglos die beiden folgenden Sätze:

Satz 5.2: Wenn $\Delta$ für $n=6, p=0$ isospektral zum Laplacian selbigen Typs einer konformflachen Mannigfaltigkeit mit verschwindender Skalarkrümmung ist, so hat auch $(\boldsymbol{M}, g)$ diese Eigenschaften.

Satz 5.3: Wenn $\Delta-\frac{1}{5} R I$ für $n=6, p=0$ isospektral zum Laplacian selbigen Typs einer konform-flachen Mannigfaltigkeit ist, so ist auch $(M, g)$ konform-flach.

Auch in der spektralen Geometrie des kanonischen Laplacians $\Delta+\varepsilon Z$ führt die Suche nach Werten $n, p$ mit definitem $U_{2}(M)$ zu Ergebnissen. Wir schlagen hier jedoch einen anderen Weg ein: Nicht die Definitheit einer gewissen quadratischen Form, sondern die Regularität einer gewissen Matrix wird ausgenutzt. 
Theorem 5.3: Wenn der kanonische Laplacian $\varepsilon \delta d-d \delta \equiv \Delta+\varepsilon Z$ für ein $n \geqq 4$ und für drei paarweise verschiedene Stufen $p=p_{1}, p_{2}, p_{3}$ isospektral zum Laplacian einer flachen Mannigfaltigkeit ist, so ist $(M, g)$ flach. Dabei sei $\Lambda^{p} T^{*}$ nur für $p \leqq \frac{n}{2}$ betrachtet.

Beweis: Aus der Formel

$$
\begin{aligned}
& N_{2}^{4} \cdot M_{2}^{p}=-\frac{1}{2} N_{1}^{2} \cdot M_{1}^{p}+N_{0}^{2} \cdot\left(M_{1}^{p}\right)^{2} \\
& \text { folgt für } n \geqq 4 \quad \operatorname{Rang}\left(1, M_{1}^{p_{i}}, M_{2}{ }^{p_{i}}\right)=\operatorname{Rang}\left(1,{M_{1}}^{p_{i}},\left(M_{1}{ }^{p_{i}}\right)^{2}\right)
\end{aligned}
$$

mit dem Matrizen-Zeilenindex $i=1,2,3$. Die Vandermondesche Matrix auf der rechten Seite ist bekanntlich genau dann regulär, wenn $M_{1}^{p_{1}}, M_{1}^{p_{2}}, M_{1}^{p_{2}}$ paarweise verschieden sind. Dann sind beide Matrizen-Faktoren in der Gleichung

$$
\left(1, M_{1}^{p_{i}}, M_{2}^{p_{i}}\right) \cdot\left(\begin{array}{lll}
1 & -1 & \frac{5}{2} \\
-15 & 90 & 30 \varepsilon \\
90(2+\varepsilon) & 360 \varepsilon & 90
\end{array}\right) \cdot\left(\begin{array}{l}
\|R i e m\|^{2} \\
\|R i c\|^{2} \\
\|R\|^{2}
\end{array}\right)=0
$$

regulär, so daß man schließen kann:

$$
\text { Spaltenvektor }=0 \Rightarrow \| \text { Riem } \|^{2}=0 \Rightarrow \text { Riem }=0
$$

Für $A T^{*}$ finden sich Aussagen ähnlichen Typs bei V. K. Рatodi [13].

\section{§ 6. Ergebnisse zum Huygensschen Prinzip}

In diesem Paragraphen setzen wir durchweg $n=6$ und lorentzsche Signatur der Metrik $g$ voraus. Im Gegensatz zur spektralen Geometrie sind jetzt Ausdrücke $\mid$ Tensor $\left.\right|^{2}$ nicht automatisch definit, und die Terme mit $\Delta R$ fallen nicht automatisch weg. Beiden Umständen muß durch zusätzliche Annahmen begegnet werden, die aber immer noch reichhaltige Klassen von Metriken einschließen werden.

Theorem 6.1: Es seien die folgenden Bedingungen erfüllt:

(1) $\quad g=g^{\prime}+g^{\prime \prime}, \quad n^{\prime} \equiv \operatorname{dim} g^{\prime} \geqq 2, \quad g^{\prime \prime}$ flach;

(2) $\quad\left|S^{\prime}\right|^{2}$ positiv definit für $n^{\prime} \geqq 3$,

$\mid W$ eyl $\left.l^{\prime}\right|^{2}$ positiv definit für $n^{\prime} \geqq 4$;

\begin{tabular}{|c|c|c|c|c|c|}
\hline $\begin{array}{c}E=\quad n^{\prime}=+ \\
+\end{array}$ & 2 & 3 & 4 & 5 & 6 \\
\hline$\otimes T^{*}$ & 2 & 4 & 6 & 10 & 15 \\
\hline$\Pi I T^{*}$ & 2 & 3 & 5 & $6-$ & 8 \\
\hline$\Pi T^{*} / g$ & 2 & 3 & 4 & 6 & 7 \\
\hline
\end{tabular}

(3) $\quad \Delta^{\prime} R^{\prime} \leqq 0$ oder $M$ wird von einer $n^{\prime \prime}$-parametrigen Schar geschlossener $n^{\prime}$-dimensionaler Untermannigfaltigkeiten $M^{\prime}$ mit $g^{\prime}$ als induzierter Metrik überdeckt;

(4) $\quad$ ist größer oder gleich der aus der Tabelle

entnommenen Schranke.

Wenn dann der reine Laplacian $\Delta$ dem $H P^{\prime}$ genügt, so ist $(M, g)$ flach. 
mit

Beweis: Aus dem $H P$ folgt unter Benutzung von Satz 4.5 und Satz 3.5:

$$
a_{2} \mid \text { Riem }\left.^{\prime}\right|^{2}+a_{1}\left|R i c^{\prime}\right|^{2}+a_{0} R^{\prime 2}+a \Delta^{\prime} R^{\prime}=0
$$

$$
\left(a_{2}, a_{1}, a_{0}, a\right)=\left(1-15 M_{1}{ }^{p},-1, \frac{5}{2}, 6\right) .
$$

Die Transformation (3.28) ergibt

$$
b_{1}=b_{2}<b_{0}=5 n^{\prime 2}-7 n^{\prime}+6-60 M_{1}{ }^{p} .
$$

Die Bedingung $b_{0}<0$ ist äquivalent zu

$$
\begin{array}{ll}
10 p>5 n^{\prime 2}-7 n^{\prime}+6 & \text { für } \otimes T^{*}, \\
10(p+3)^{2}>45 n^{\prime 2}-49 n^{\prime}+132 & \text { für } \Pi T^{*}, \\
2(p+2)^{2}>5 n^{\prime 2}-7 n^{\prime}+14 & \text { für } \Pi T^{*} / g,
\end{array}
$$

und dies ist - wie man leicht nachrechnet - wiederum äquivalent zu (4). Nimmt man noch die Bedingungen (2) und $\Delta^{\prime} R^{\prime} \leqq 0$ hinzu, so wird die linke Seite von (6.1) negativ definit, und wir können schließen:

$$
\begin{aligned}
& R^{\prime}=0, \quad S^{\prime}=0 \text { für } n^{\prime} \geqq 3, \text { Weyl } l^{\prime}=0 \text { für } n^{\prime} \geqq 4 \\
& \Rightarrow \text { Riem }=0 \Rightarrow \text { Riem }=0 .
\end{aligned}
$$

Nimmt man anstelle von $\Delta^{\prime} R^{\prime} \leqq 0$ die Bedingung mit den Untermannigfaltigkeiten $M^{\prime}$ hinzu, so integriere man (6.1) über die $M^{\prime}$ und behandle

$$
a_{2}\left\|R_{i e m}^{\prime}\right\|^{2}+a_{1}\left\|R^{\prime} c^{\prime}\right\|^{2}+a_{0}\left\|R^{\prime}\right\|^{2}=0
$$

auf entsprechende Weise weiter

Theorem 6.2: Es seien $|S|^{2}, \mid$ Weyl $\left.\right|^{2}$ positiv definit. Wenn dann der Laplacian mit verschwindender Cottoninvariante $\Delta-\frac{1}{5} R I$ für ein $p \geqq 1$ dem $H P$ genügt, so ist $(M, g)$ flach.

Beweis: Die in $§ 5$ für definite Metriken ausgeführten Schlüsse können sinngemäß auf

$$
a_{2} \mid \text { Riem }\left.\right|^{2}+a_{1}|R i c|^{2}+a_{0} R^{2}=0
$$

mit

$$
\left(a_{2}, a_{1}, a_{0}\right)=\left(1-15 M_{1}^{p},-1, \frac{1}{10}\right)
$$

übertragen werden, wobei jetzt $n=6, p \geqq 1$ I

$\mathrm{Zu}$ den beiden Theoremen sind noch einige Bemerkungen angebracht:

1. Theorem 6.2 ist so gemeint, daß $E=\Lambda T^{*}$ mit erfaßt ist; bei Theorem 6.1 ist dagegen $\Lambda T^{*}$ nicht mit erfaßt. Die Aussage von Theorem 6.1 gilt aber sinngemäß auch noch, wenn man anstelle der Bedingung (4) einsetzt:

$$
E=\Lambda T^{*} ; n^{\prime}=2 ; p=2,3,4 .
$$

2. Die Definitheitsbedingungen in den Theoremen 6.1, 6.2 erscheinen zunächst recht „speziell“. Wir können aber einfach zu handhabende Klassen von Metriken 
angeben, welche diese Bedingungen erfüllen:

Nach Satz 3.3 gilt:

$g$ statisch $\Rightarrow|S|^{2},|W e y l|^{2}$ positiv definit;

$g^{\prime}$ statisch und $n^{\prime} \geqq 3 \Rightarrow\left|S^{\prime}\right|^{2}$ positiv definit;

$g^{\prime}$ statisch und $n^{\prime} \geqq 4 \Rightarrow\left|W e y l^{\prime}\right|^{2}$ positiv definit.

Außerdem gilt natürlich :

$$
\begin{aligned}
& g^{\prime} \text { definit und } n^{\prime} \geqq 3 \Rightarrow\left|S^{\prime}\right|^{2} \text { positiv definit; } \\
& g^{\prime} \text { definit und } n^{\prime} \geqq 4 \Rightarrow\left|W e y l^{\prime}\right|^{2} \text { positiv definit. }
\end{aligned}
$$

(Man bemerkt hier noch: $g^{\prime}$ definit $\Rightarrow g^{\prime \prime}$ lorentzsch, $g^{\prime}$ statisch $\Rightarrow g^{\prime \prime}$ definit.)

3. Für $p=0$ ergibt sich eine Nullstelle von $b_{1}$ bzw. von $b_{1}, b_{0}$ gleichzeitig:

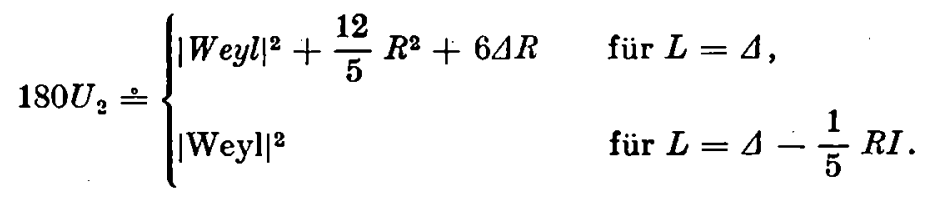

Aus der letzten Bemerkung ergeben sich zwanglos die beiden folgenden Sätze.

Satz 6.1: Es sei $\mid W$ eyl $\left.\right|^{2}$ positiv definit und ( $\Delta R \geqq 0$ oder $M$ geschlossen). Wenn dann $\Delta$ für $p=0$ dem $H P$ genügt, so ist $(M, g)$ konform-flach und hat verschwindende Skalarkrümmung.

Satz 6.2: Es sei $\mid$ Weyl $\left.\right|^{2}$ positiv definit. Wenn dann $\Lambda-\frac{1}{5}$ RI für $p=0$ dem $H P$ genügt, so ist $(\boldsymbol{M}, g)$ konform-flach.

$\mathrm{Zu}$ den in Bemerkung 2 angesprochenen statischen Metriken sind die retardierten Metriken verwandt (siehe $\S 3$, insbesondere Satz 3.4). Für diese kann man Aussagen von der Bauart

$$
L \text { huygenssch } \Rightarrow g \text { ist } p p \text {-Wellen-Metrik speziellen Typs }
$$

erhalten:

Satz 6.3: Es sei $g$ retardiert und $\Delta R \leqq 0$ oder $M$ geschlossen und $p$ sei größer oder gleich der aus der Tabelle

\begin{tabular}{l|c}
\hline$\otimes T^{*}$ & 15 \\
\hline$\Pi T^{*}$ & 8 \\
\hline$\Pi T^{*} / g$ & 7 \\
\hline
\end{tabular}

entnommenen Schranke. Wenn dann $\Delta$ dem HP genügt, so ist $g$ eine $p p$-Wellen-Metrik vom Robinsonschen Typ (3.22).

Der Beweis verläuft bis zu der Stelle

$$
\mid \text { Riem }\left.\right|^{2}=\mid \text { Ric }\left.\right|^{2}=R^{2}=0
$$

analog zu dem von Theorem 6.1 für den Fall $n^{\prime}=6$. Weiter wird mit den Sätzen 4.7 und 3.4 geschlossen:

$$
0=12 U_{2}(x, x)=K_{\alpha \beta} K^{\alpha \beta} \Rightarrow R_{\alpha \beta \mu \nu} R^{\alpha \beta \mu} \lambda=0 \Rightarrow \text { Behauptung }
$$



Satz 6.4: Es sei $g$ retardiert und $E^{p} \neq \Lambda^{3} T^{*}$. Wenn dann $\Delta-\frac{1}{5} R I$ dem $H P$
genügt, so ist $g$ eine $p p$-Wellen-Metrik vom Robinsonschen Typ (3.22).

Der Beweis verläuft bis zu der Stelle

$$
\mid \text { Riem }\left.\right|^{2}=\mid \text { Ric }\left.\right|^{2}=R^{2}=0
$$

analog zu dem von Theorem 6.2. Weiter wird wie beim Beweis von Satz 6.3 geBchlossen

Satz 6.5: Es sei $g$ retardiert und $E^{p}=\Lambda^{3} T^{*}$. Wenn dann $\Delta-\frac{1}{5} R I$ dem $H P$ genügt, so ist $g$ eine $p p$-Wellen-Metrik vom Kundtschen Typ (3.20).

Beim Beweis kann nur Satz 3.4, nicht aber Satz 4.7 angewendet werden, da jetzt gerade der Ausnahmefall $E^{p}=\Lambda^{\frac{n}{2}} T^{*}$ vorliegt

Die beim Beweis von Theorem 5.3 angewendeten Schlußweisen können sinngemäß auf das Problem des HP übertragen werden:

Theorem 6.3: Es sei $\mid$ Riem $\left.\right|^{2}$ definit und $(\Delta R=0$ oder $M$ geschlossen). Wenn dann der kanonische Laplacian $\varepsilon \delta d-d \delta \equiv \Delta+\varepsilon Z$ für drei paarweise verschiedene Stufen $p=p_{1}, p_{2}, p_{3}$ dem $H P$ genügt, so ist $(M, g)$ flach. Dabei sei $\Lambda^{p} T^{*}$ nur für $p=0,1$, 2, 3 betrachtet.

Die vorliegende Arbeit wurde von Herrn Prof. Dr. P. Günther gefördert und ist aus dem von ihm geleiteten Seminar hervorgegangen. Der Autor möchte ihm hiermit seinen Dank aussprechen. Herrn Dipl.-Math.W. QUAPP sei für einige Kontrollrechnungen gedankt und Herrn Dr. J. ErchноRN für nützliche Hinweise.

\section{LITERATUR}

[1] BeL, L.: La radiation gravitationelle. In: Colloque do Royaumont 1959. Ed. CNRS: Paris 1962.

[2] Berger, M.: Le spectre des variétés Riemanniennes. Rev. Roumaine Math. Phys. Appl. 13 (1968), 915-931.

[3] Courant, R., und D. Hmbert: Methoden der mathematischen Physik. Springer-Verlag: Berlin 1968.

[4] GÆKey, P. B.: Curvature and the eigenvalues of the Laplacian for elliptic complexes. Advances Math. 10 (1973), $344-382$.

[5] Grokey, P. B.: The spectral geometry of real and complex manifolds. AMS Proc. Symp. Pure Math. 27 (1975), 265-280.

[6] Günther, P.: Ubber die Darbouxsche Differentialgleichung mit variablen Koeffizienten. Math. Nachr. 22 (1960), 285-321.

[7] Günther, P.: Einige Sütze über huygenssche Differentialgleichungen. Wiss. Z. KarlMarx-Univ. Leipzig, Math.-nat. R. 14 (1965), $497-507$.

[8] Günther, P., and R. Schmmma: Curvature and Spectrum of Compact Riemannian Manifolds. J. Diff. Geom. 12 (1977), 599-618.

[9] HADAMARD, J.: Lectures on Cauchy's problem in linear partial differential equations. Yale University Press: New Haven 1923.

[10] Hall, G. S.: The classification of the Ricci tensor in general relativity theory. J. Phys. A 9 (1976), 541-545.

[11] McKean, H. P., Jr., and I. M. SnNGer: Curvature and the eigenvalues of the Laplacian. J. Diff. Geom. 1 (1967), 43-69.

[12] Kramer, D., H. Stephani, and M. A. H. Macoallom: Exact solutions of Einstein's field equations. VEB Deutscher Verlag der Wissenschaften, Berlin 1980. 
[13] Patodi, V. K.: Curvature and the eigenforms of the Laplace operator. J. Diff. Geom. 5 (1971), 233-249.

[14] Schmming, R.: Riemannsche Metriken mit ebener Symmetrie und das Huygenssche Prinzip. Dissertation A. Leipzig 1971.

[15] Schmomng, R.: Riemannsche Räume mit ebenfrontiger und mit ebener Symmetrie. Math. Nachr. 59 (1974), $129-162$.

[16] Schimarna, R.: Bedingungen reiner Strahlung in der allgemeinen Relativitätstheorie. Wiss. Schriftenr. TH Karl-Marx-Stadt 1975, 5. TMP Heft 3, 424-432.

[17] Sormiming, R.: Lineare Differentialoperatoren zweiter Ordnung mit metrischem Hauptteil und die Methode der Koinzidenzwerte in der Riemannschen Geometrie. Beiträge zur Analysis 15 (1981), 77-91.

[18] Scrimming, R.: Cauchyproblem und Wellenlösungen der Bachschen Feldgleichungen der allgemeinen Relativitätstheorie. Dissertation B. Leipzig 1979

[19] Trautman, A.: Foundations and current problems of general relativity. In: Lectures on general relativity, Brandeis summer institute in theoretical physics, Prentice-Hall, Englewood Cliffs, New York 1965.

[20] Щиммивг, Р.: Гюигенсовы гиперболические уравнения второго порядка для многокомпонентных полей. Укр: мат. ж. 29-(1977), 351-363.

Manuskripteingang: 20. 03.1981

VERFASSER:

Dr. sc. RATNer SoBtmarno

Sektion Mathematik der Ernst-Moritz-Arndt-Universität

DDR-2200 Greifswald, Friedrich-Ludwig-Jahn-Str. 15a 